2. To: (Receiving Organization)

Characterization Project Operations (75100)

5. Proj./Prog./Dept./Div.: Core Sampling / FGWL Tanks

8. Originator Remarks:

ETN-96-003

This report documents the completion of the formal design review for the Rotary Mode Core Sample Truck (RMCST) modifications for flammable gas tanks. The RMCST modifications are intended to support core sampling operations in waste tanks requiring flammable gas controls. 11. Receiver Remarks:

3. From: (Originating Organization)

Characterization Equipment Design (75230)

6. Cog. Engr.:

R. H. Stubbs
7. Purchase Order No.:

$\mathrm{N} / \mathrm{A}$

9. Equip./Component No.:

$\mathrm{N} / \mathrm{A}$

10. System/Bldg./Facility:

\section{Genera1}

12. Major Assm. Dwg. No.: $N / A$

13. Permit/Permit Application No.: N/A

14. Required Response Date: $5 / 29 / 96$

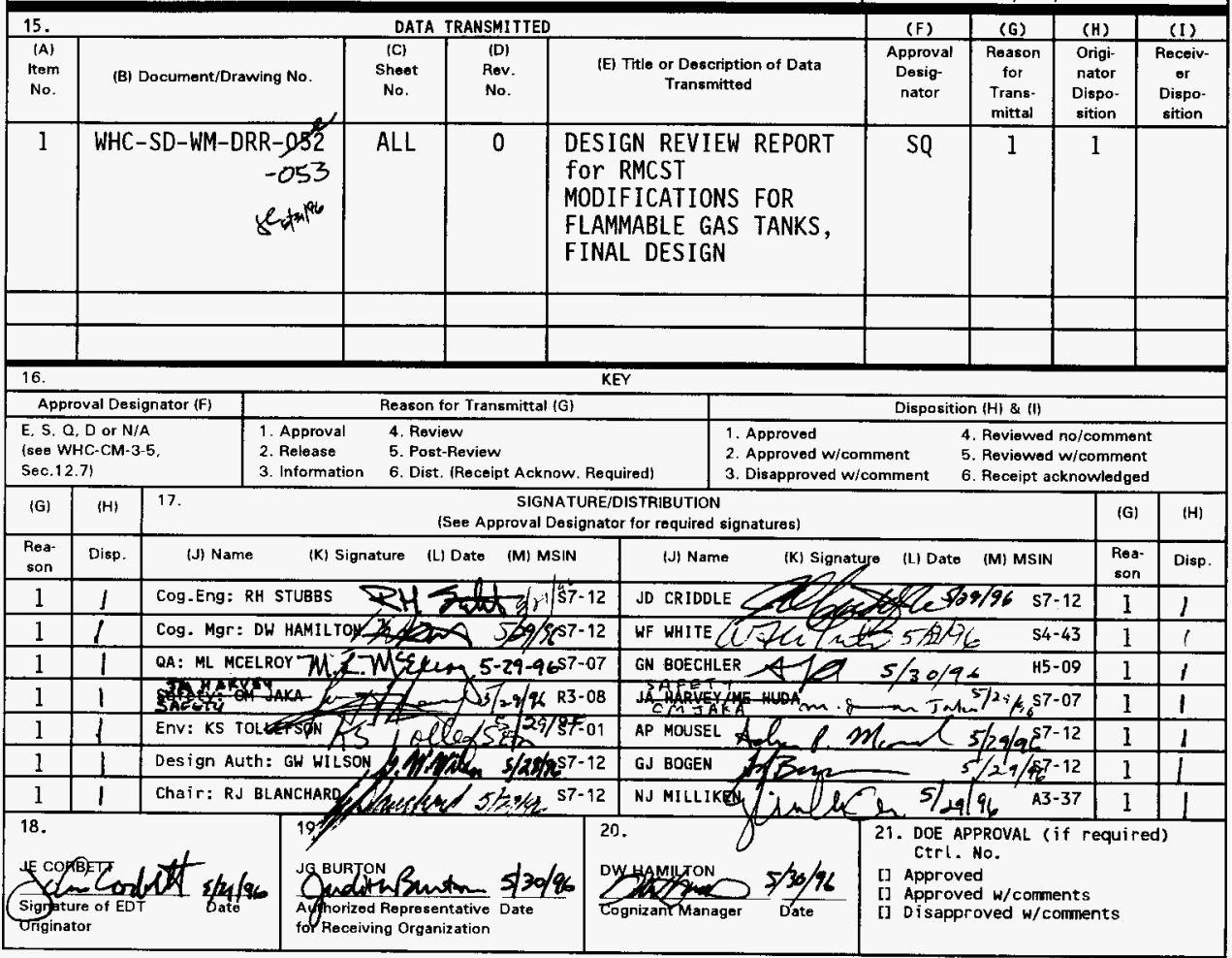




\title{
FINAL DESIGN REVIEW REPORT for RMCST MODIFICATIONS FOR FLAMMABLE GAS TANKS
}

\author{
J. E. Corbett \\ Westinghouse Hanford Company, Richland, WA 99352 \\ U.S. Department of Energy Contract DE-AC06-87RL10930 \\ EDT/ECN: 615409 \\ Org Code: W75230 \\ B\&R Code: $35=2131207$ \\ UC: 2030 \\ Charge Code: N4HBB \\ Total Pages: $\% \%$
}

Key Words: RMCST modifications, Core Sampling, Flammable Gas Watch List, Rotary Mode Core Sampling, RMCS, Core Sample Truck, Final Design Review, Formal Design Review, Design Review Report

Abstract: This report documents the completion of the formal design review for the Rotary Mode Core Sample Truck (RMCST) modifications for flammable gas tanks. The RMCST modifications are intended to support core sampling operations in waste tanks requiring flammable gas controls. The objective of this review was to approve Engineering Change Orders and new drawings, at the 100\% design completion state. The conclusion reached by the review committee was that the design was acceptable and efforts should continue toward fabrication and delivery.

TRADEMARK DISCLAIMER. Reference herein to any specific commercial product, process, or service by trade name, trademark, manufacturer, or otherwise, does not necessarily constitute or imply its endorsement, recommendation, or favoring by the United States Govermment or any agency thereof or its contractors or subcontractors.

Printed in the United States of America. To obtain copies of this document, contact: WHC/BCS Document Control Services, P.O. Box 1970, Mailstop H6-08, Richland WA 99352, Phone (509) 372-2420; Fax (509) 376-4989.

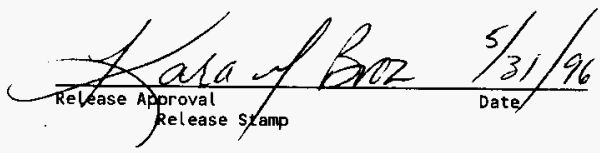

Approved for Public Release 
WHC-SD-WM-DRR-053, Rev. 0

\section{FINAL DESIGN REVIEW REPORT}

for

RMCST Modifications for Flammable Gas Tanks

Issued by:

J. E. Corbett, Senior Engineer

Tank Waste Remediation System

Characterization Project 


\section{TABLE OF CONTENTS}

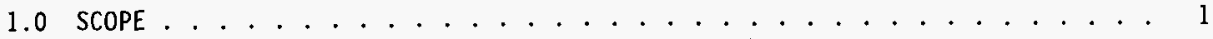

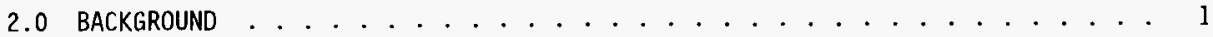

3.0 SUMmarY . . . . . . . . . . . . . . . . . . . . . . . . 2

4.0 GENERAL DESIGN DESCRIPTION . . . . . . . . . . . . . . . . . . 2

5.0 MODIFICATION DESIGN DESCRIPTION . . . . . . . . . . . . . . . . . . . . 3

6.0 LiSTING OF REVIEW MATERIALS . . . . . . . . . . . . . . . . . . . . . . . . 5

7.0 SA COMPLIANCE MATRIX . . . . . . . . . . . . . . . . . . . . . . . . 6

8.0 DESIGN REVIEW CHECKLIST . . . . . . . . . . . . . . . . . . . . 8

9.0 REVIEW COMMENT RECORDS . . . . . . . . . . . . . . . . . . . . . . 8

10.0 CONCLUSIONS AND OPEN ITEMS . . . . . . . . . . . . . . . . . . . . . . . . . 9

11.0 DOCUMENTATION . . . . . . . . . . . . . . . . . . . . . . . . 10

\section{ACRONYMS}

$\begin{array}{ll}\text { ATP } & \text { acceptance test procedure } \\ \text { CED } & \text { Characterization Equipment Engineering } \\ \text { CFR } & \text { Code of Federal Regulations } \\ \text { CPO } & \text { Characterization Project Operations } \\ \text { CST } & \text { core sample truck } \\ \text { DRR } & \text { design review report } \\ \text { ECN } & \text { engineering change order } \\ \text { EP } & \text { Engineering Practices } \\ \text { FDC } & \text { functional design criteria } \\ \text { FGWL } & \text { flammable gas watch list } \\ \text { HEPA } & \text { high efficiency particulate air (filter) } \\ \text { NEC } & \text { National Electrical Code } \\ \text { NFPA } & \text { National Fire Protection Association, Inc. } \\ \text { OTP } & \text { operability test procedure } \\ \text { RCR } & \text { review comment record } \\ \text { RLU } & \text { remote latch unit } \\ \text { RMCS } & \text { rotary mode core sampling } \\ \text { RMCST } & \text { rotary mode core sampling truck } \\ \text { WHC } & \text { Westinghouse Hanford Company }\end{array}$




\section{RMCST Modifications for Flammable Gas Tanks, Fina) Design Review}

\subsection{SCOPE}

This report documents the completion of the formal design review for Rotary Mode Core Sample Truck modifications for flammable gas tanks, hereafter referred to as the "the RMCST modifications." The RMCST modifications are intended to support core sampling operations in waste tanks requiring flammable gas controls. This review included preliminary and final review of new drawings, and engineering change orders ( $E C N^{\prime} s$ ) for existing drawings, considered to be at the $100 \%$ design completion state. These documents are listed in section 6.0 .

This document and the formal design review are in support of design modifications to the core sampling systems used by Characterization Project Operations (CPO). The RMCST modifications are required to expand the scope of core sampling to include Flammable Gas Watchlist (FGWL) tanks, as well as any other tanks with flammable gas controls. The objective of this review was to provide a formal design verification consisting of a systematic overall review and evaluation of the RMCST flammable gas modifications. Design verification is performed to insure equipment function, personnel safety, and compliance with WHC-CM-6-1, Standard Engineering Practices, section 2.2.4, and the quality assurance requirements of 10 CFR 830.120 . Acceptance of the design is required prior to operational use on any waste tanks requiring flammable gas controls. RMCS exhauster modifications and the hydrogen interlock design are evaluated in separate design reviews and are not part of this report.

\subsection{BACKGROUND}

The Rotary Mode Core Sampling (RMCS) systems were designed for initial deployment to FeCN tanks, with later modifications intended to allow for deployment to Flammable Gas Watch List (FGWL) tanks. These modifications include electronic components designed for intrinsic safety, additional nitrogen purge components and controls, and part replacement where required for material compatibility with hazardous environments. The modifications include replacing or adding equipment to ensure that electrical components which are not classified for use in flammable atmospheres are isolated from potentially flammable atmospheres. This was accomplished by replacing these components with components approved for use in flammable atmospheres, and controlling the potential for flammable atmospheres where possible to isolate non classified electrical components from the hazard. The modifications also include part replacement where required for material compatibility associated with hazardous environments. A functional design criteria (WHC-SD-WM-FDC-048) was used to establish the basis criteria for the modifications. 


\subsection{SUMMARY}

The design review committee was selected in accordance with EP 4.1 and is documented in Section 11.0 of this report. A preliminary design review was performed at the $30 \%$ and $50 \%$ levels. This portion of the formal design review was completed on February 5th, 1996, and is documented in WHC-SD-WM-DRR-052.

The kickoff meeting for the $100 \%$ level design review was held on February 6th, 1996. This meeting served as the first of two final design review briefings. Updated design information and the majority of the modification work ECN's were provided at this briefing. The second final design review briefing was held on February 22nd, where a majority of the new drawings were provided for review. Meeting minutes for these briefings are attachments to Section 11.0 of this report.

The final design review close-out meetings were delayed in order to incorporate changes made to the SA, WHC-SD-WM-SAD-035, A Safety Assessment of Rotary Mode Core Sampling in Flammable Gas Single Shell Tanks. The first close-out meeting was held on April 25th. Fifteen ECN's and four new drawings were approved. Of the twenty-three review comment records (RCR's) submitted for this meeting, twenty-one were closed out, and two were withdrawn or superseded. The test results for the mechanical remote latch unit (RLU) were provided (refer to WHC-SD-WM-TR-032). The second of the two close-out meetings was held on May 7 th. Two additional ECN's and the remaining two drawings were approved. Six RCR's were submitted and closed out at, or prior to, this meeting. One RCR was submitted after the close-out meetings to document a requested change to the shielded receiver hoist switch. This RCR remains an open item. For a complete listing of RCR's, see section 9.0 of this report. A listing of open items can be found in section 10.0. Meeting minutes for the close-out meetings, and the completed design review checklist, are attachments to Section 11.0 of this report.

After the completion of the close-out meetings, the conclusion reached by the review committee was that the design of the RMCST modifications was acceptable and efforts should continue toward fabrication and delivery.

\subsection{GENERAL DESIGN DESCRIPTION}

In response to Characterization Program sampling schedule needs, modifications to existing core sampling equipment were kept to a minimum where practical. Changes were made primarily to equipment that can be exposed to potentially flammable gases during sampling of FGWL tanks, including the drill bit, drill string, grapple box, remote latch unit, and shielded receiver enclosures. New equipment and modifications were designed to interface with existing core sampling equipment.

The existing RMCST is designed to sample liquid, sludge, and/or salt cake materials in waste tanks, using the universal sampler, while operating in the outdoor environment of the tank farms. RMCST interfaces are limited to tank riser flanges and RMCS system internal interfaces. The truck is positioned in the tank farms and sections of drill rod are assembled and lowered through the riser to the tank waste level. The assembly of drill rod sections is known as the drill string, which is connected to, and controlled by, the drill rig located on the core sample truck. Using the RLU and shielded receiver hoist, the sampler is lowered down through the drill string and seals above the driil 
bit, in the core barrel. The Quadralatch portion of the sampler locks the sampler into place and rotates with the drill string while the sample section stays stationary. When the drill penetrates and the sampler descends, a piston in the sampler is held in place by the grapple assembly, allowing the core sample to be captured within the sampler. The sampler is designed to prevent tank waste from entering the drill string. Seal failure would expose the drill string and grapple box to tank waste/waste gases. For RMCS in flammable gas tanks, the drill string and grapple box volumes are classified as Nationa1 Fire Protection Association (NFPA) Class 1, Division 1, Group B flammable hydrogen atmospheres.

After a 19-inch core sample segment is taken, a valve closes at the opening of the sampler, and the drill string is disconnected from the drill rig. The shielded receiver, also located on the core sample truck, is then connected to the drill string and is used to retrieve the sample and install a new sampler. Prior to reconnecting the drill string to the drill rig, the length of the drill string is increased by installing an additional 19-in. threaded segment to prepare for the next sampling cycle. During sampler change-out, waste is prevented from entering the drill string by establishing a hydrostatic head balance. Failure of the hydrostatic head balance would expose the drill string and the shielded receiver to tank waste/waste gases. For RMCS in flammable gas tanks, the shielded receiver tube and shielded receiver box (sampler hoist housing) volumes are classified as NFPA Class 1, Division 1, Group B flammable hydrogen atmosphere. A seal failure on the shielded receiver box would expose equipment under the weather cover to gasses from the shielded receiver. For RMCS in flammable gas tanks, the volume within the shielded receiver weather cover, external to the shielded receiver box, is classified as a NFPA Class 1, Division 2, Group B flammable hydrogen atmosphere.

In addition to the core sample truck, the RMCS systems include the nitrogen supply trailer, used to provide the nitrogen gas for establishing hydrostatic head balance during sampler changeout, and to supply nitrogen purge gas to cool the drill bit and clear away the cuttings during drilling. For tanks which do not have an operating exhauster, the RMCS exhauster is set up to filter and remove this additional gas volume to comply with tank emissions regulations and safety requirements. The RMCS trucks have an exhauster interlock system to shut down the drill rig whenever the RMCS exhauster is shut down. The RMCS systems also includes ancillary equipment to supply electrical power, power distribution, equipment wash water, lighting, breathing air, communication, and equipment storage, transportation and handling.

\subsection{MODIFICATION DESIGN DESCRIPTION}

The stainless steel riser sleeve provided for RMCS in flammable gas tanks is required to reduce the risk of spark initiation from contact between the carbon steel riser and the rotating carbon steel drill string. The riser sleeve assembly includes a spray wash system which routes pressurized wash water from a coupling at the sleeve flange to spray nozzles located at the bottom of the sleeve. This feature will be used during drill string removal to wash the $O D$ of the lower drill string sections before they are raised through the sleeve. The existing wash water hose from the RMCS support truck (previously designed to connect to the drill rod washer) will interface with the connection on the riser sleeve flange. 
A riser sleeve spray washer is installed between the riser flange (or off set riser adapter) and the riser sleeve flange. The spray washer will be used during riser sleeve removal to reduce contamination on the sleeve exterior. A smaller riser adapter is provided to compensate for the added height of the sleeve and spray washer. New offset riser adapters are provided to accommodate clearance needed between the riser sleeve and the tank riser.

A nitrogen gas purge is provided to the riser sleeve to prevent flammable gas accumulation in the riser sleeve. Nitrogen gas is delivered by the RMCS nitrogen trailer, through the core sample truck purge gas piping system, to a new hose reel located at the back of the core sample truck. The new hose reel and pneumatic hose supply nitrogen to the riser sleeve through a coupling on a modified drill rod washer. The nitrogen flows down through riser sleeve into the tank dome space. The purge gas pneumatic diagram is shown on $\mathrm{H}-2-690009$ and ECN 628717. Flow control for riser liner purge consists of a ball valve and flow control valve, located at the rear of the core sample truck, below the rotating platform.

The dri1l rod washer is modified to prevent inadvertent connection to the core sample truck "vent to tank" hose. The coupling size is changed to accept connection to the nitrogen hose coupling from the new hose reel, for the purpose of riser liner purge.

Nitrogen purge is added to the shielded receiver weather cover to comply with NFPA Article 496, Type $Z$ purging requirements of enclosures for use in flammable hydrogen atmospheres. The shielded receiver weather cover is supplied with nitrogen from the RMCS nitrogen trailer, through the core sample truck purge gas piping system. The nitrogen purge under the shielded receiver weather cover is continually vented to atmosphere through the HEPA filter installed on the cover. A pressure relief valve is added to the weather cover to assure that Z-Purge pressure cannot build up and damage the weather cover if the HEPA filters become plugged. The purge gas pneumatic diagram is shown on $\mathrm{H}-2-690009$ and ECN 628717. Flow control for z-purge consists of a metering valve, located on the shielded receiver lifting frame.

A pressure switch and pressure differential switch are added to monitor the nitrogen gas purge in the shielded receiver weather cover and tank riser liner (respectively). Electrical signals are sent to the instrument enclasure assembly where alarm lights are located. The purge gas flow and pressure requirements are documented in the SA. During rotary mode core sampling, a loss of riser liner purge gas, defined as less than 40 psi differential pressure, will cause an automatic shutdown of the dri11 rig.

Intrinsic safety barriers are added to the shielded receiver and grapple loadcells. These electrical modifications do not effect operator interface. The barriers are necessary to meet the requirements of NFPA Class 1, Division 1 , Group B, flammable hydrogen atmosphere.

The original electrically operated remote latch unit (RLU) is replaced with a new mechanical RLU. Because the mechanical RLU does not require any electrical power, it can be operated in the potentially flammable gas atmosphere of the dri11 string. The latching control chassis is deleted from the control console, and the slip ring and cable wiring are deleted from the hoist, since the remote latch unit electronic latching controls are not needed for the mechanical RLU. Operator actions required to control the mechanical RLU are limited to hoist control. 
Penetration rate alarms are added to the critical alarm system. The alarm is activated when the penetration rate of the drill head is outside of acceptable limits. Instrumentation is located on the drill head and the digital display is located in the instrument enclosure assembly.

Set points and delay times are changed for other critical alarms to comply with envelope parameters for sampling in flammable gas tanks. Alarm functions are controlled by the PLC, which is reprogrammed as part of these modifications. Set points and delay times are listed in the SA.

\subsection{LISTING OF REVIEW MATERIALS}

The following ECN's were reviewed and approved as part of the formal design review:

\begin{tabular}{|c|c|}
\hline ECN \# & DESCRIPTION \\
\hline 623775 & $\begin{array}{l}\text { shows changes in the control logic of core Sample Truck } \# 3 \& 4 \text {, to implement flammable gas } \\
\text { modifications }\end{array}$ \\
\hline 626740 & $\begin{array}{l}\text { adds PENETRAIION RATE alarms to the Instrument enclosure assembly drawing, electrical } \\
\text { connection diagram and elementary diagram of Core Sample Trueks } \# 3 \text { and } \# 4\end{array}$ \\
\hline 626741 & $\begin{array}{l}\text { pressure switches are added to monitor purge gas pressure in the shielded receiver weather } \\
\text { cover and tank riser liner }\end{array}$ \\
\hline 626742 & $\begin{array}{l}\text { adds intrinsically safe barriers to the loadcell functions to the electrical connection } \\
\text { diagram and elementary diagram of core Sample Trucks } \# 3 \text { and } \# 4 \text {; removes Latching Control } \\
\text { chassis }\end{array}$ \\
\hline 628706 & $\begin{array}{l}\text { adds } z \text {-Purge to the shielded receiver weather cover and adds an additional hose reel and } \\
\text { associated piping to provide riser liner purge capabilities }\end{array}$ \\
\hline 628707 & $\begin{array}{l}\text { adds an intrinsic safety barrier to the load cell for the shielded receiver assembly to } \\
\text { allow sampling flammable gas watchlist tanks }\end{array}$ \\
\hline 628708 & $\begin{array}{l}\text { changes the shielded receiver base assembly to add required penetrations for addition of } \\
\text { filter, Z-Purge lines and MS connectors for intrinsic safety barrier }\end{array}$ \\
\hline 628709 & $\begin{array}{l}\text { deletes slip ring on the sampler hoist since it is no longer needed because the remote latch } \\
\text { unit is being replaced with a mechanical remote latch unit to allow sampling in flamable } \\
\text { gas watchlist tanks }\end{array}$ \\
\hline 628710 & $\begin{array}{l}\text { adds the mechanical remote latch unit, the riser liner, riser liner spray washer, riser } \\
\text { adapter, and riser arrangement drawings to drawing index }\end{array}$ \\
\hline 628711 & $\begin{array}{l}\text { changes upper assembly views to show the addition of the intrinsic safety barriers, z-Purge, } \\
\text { and riser liner purge }\end{array}$ \\
\hline 628712 & adds a mounting bracket for the intrinsic safety barrier for the grapple hoist load cell \\
\hline 628713 & $\begin{array}{l}\text { changes the weather cover to add pressure relief valve to assure that } Z \text {-Purge pressure } \\
\text { cannot build up and damage weather cover if HEPA filters become plugged (see ECN } 628706 \text { for } \\
Z \text {-Purge installation) }\end{array}$ \\
\hline 628714 & $\begin{array}{l}\text { deletes the latching control chassis from the control console since the remote latch unit is } \\
\text { being replaced with a mechanical remote latch unit to allow sanipling flamable gas watchtist } \\
\text { tanks. }\end{array}$ \\
\hline 628715 & $\begin{array}{l}\text { deletes the latching control components since the remote latch unit is being replaced with a } \\
\text { mechanical remote latch unit }\end{array}$ \\
\hline 628717 & $\begin{array}{l}\text { changes the purge gas pneunatic diagran to add the z-Purge, riser liner purge to allow } \\
\text { sampling in flamable gas watchlist tanks }\end{array}$ \\
\hline 628720 & $\begin{array}{l}\text { New assembly required for mechanical remote latch unit, required for sampling in flammable } \\
\text { gas watchlist tanks }\end{array}$ \\
\hline 630017 & ngs to accommodate riser liner purge \\
\hline
\end{tabular}


The following new drawings were reviewed and approved as part of the formal design review:

\begin{tabular}{||l|l|}
\hline DRAWING \# & \multicolumn{1}{|c|}{ TITLE } \\
\hline H-2-690128 & RISER SLEEVE ASSEMBLY \\
\hline$H-2-690131$ & RISER SLEEVE SPRAY WASHER ASSEMBLY \\
\hline$H-2-690132$ & RISER ADAPTER ASSEMBLY \\
\hline$H-2-690134$ & DRILL STRING ARRANGEMENTS (RMCST) \\
\hline$H-2-690136$ & OFFSET RISER ADAPTER 4"X 6, " 8" \& 12" \\
\hline$H-2-690142$ & MECHANICAL REMOTE LATCH UNIT \\
\hline
\end{tabular}

\subsection{SA COMPLIANCE MATRIX}

The table on the following page was used as a tool for determining which safety requirements from the SA (WHC-SD-WM-SAD-035) are design criteria for the design modifications being reviewed. Each credited design safety feature listed in the $S A$ is shown on the left side of the table. The corresponding implementing document(s), shown on the left side of the table, is generally the ECN or new drawing which incorporates the safety feature into the RMCS design for flammable gas tanks. In many cases, where the implementing document is shown as "existing," the design feature was incorporated in the existing design for the RMCS system, and no modification is required. Since the table applies to the entire RMCS system, only the core sample truck safety features are applicable to this design review. Other design reviews use the same table, as applicable to other components of the RMCS system. At the time of the final design review close-out meeting, item \#8, was considered "in work, " and the test report number associated with item \#12 was "TBD." Both of these items are discussed in section 10.0 of this report. 
SAFETY ASSESSMENT COMPLIANCE MATRIX

\begin{tabular}{|c|c|c|}
\hline$\#$ & CHAPTER 6 - SAFETY FEATURES & IMPLEMENTING DOCUMENT \\
\hline 1 & Material compatibility (On-site) & ECN 631116, 631126, H-2-690142, memo 75230-96-001, rev. 2 \\
\hline 2 & Spark-resistant tools (NA) & Administrative Control (Procedure) \\
\hline 3 & Grounding and bonding (NA) & ECN 626742 and approved grounding procedure \\
\hline 4 & Radiological controls (NA) & Existing - Exhauster housing $<100 \mathrm{mR}$ on contact \\
\hline 5 & Riser sleeve (Off-site) & ECN $628706(\mathrm{H}-2-690128, \mathrm{H}-2-690131)$ \\
\hline 6 & Drill string spray washer (NA) & Existing \\
\hline 7 & Frisbee/DS interface lubricant (NA) & Existing \\
\hline 8 & Pneumatic foot clamp (Off-site) & In work \\
\hline 9) & Drill bit configuration \& mat'l (Off-site) & Existing - certified by USBM testing \\
\hline 10 & Drill centering spike (NA) & Existing \\
\hline 11. & Chevron seal between drill bit \& sampler (NA) & Existing \\
\hline 12 & Core sampler and drill string components (Off-site) & \#TBD \\
\hline 13 & Sniffing ports (NA) & Existing (H-2-826513) \\
\hline 14 & Change-out assembly (NA) & Existing \\
\hline 15 & Cable spray washer (NA) & Existing \\
\hline 16) & Purge flow limitation (Off-site) & Existing \\
\hline 17 & Original speed limitation (Off-sife) & Existing \\
\hline 18) & Downforce limitation (Off-site) & Existing \\
\hline 19 & Drill string penetration rate (Off-site) & ECN 626740 \\
\hline 20 & Hydraulic bottom detector (Off-site) & Existing \\
\hline 21 & Walkdown function (Off-site) & Existing \\
\hline 22 & Hydrostatic head (NA) & Existing \\
\hline 23 & Truck position (Off-site) & Existing \\
\hline 24 & Stabilizing jacks (NA) & Existing \\
\hline 25 & Quill rod adapter (Off-site) & ECN 631126 \\
\hline 26 & Grapple hoist assembly (Off-site) & ECN 628712,626742 \\
\hline 27 & Grapple (sample actuator) (Off-site) & Existing \\
\hline 28 & Grapple insertion (NA) & Existing \\
\hline 29 & Grapple hoist cable tension (NA) & Existing \\
\hline 30 & Shielded receiver assembly (Off-site) & ECN $626742,628707,628713$ \\
\hline 31 & SR tube (NA) & Existing \\
\hline 32 & SR view port (NA) & Existing \\
\hline 33 & SR hoist cable tension (NA) & Existing \\
\hline 34 & Remote latch unit (Off-site) & H-2-690142, ECN $628708,628711,628714,628715$ \\
\hline 35 & RLU insertion (NA) & Existing \\
\hline 36 & RLU position indicator (NA) & Existing \\
\hline 37 & Exhauster Operation (Off-site) & ECN 632390 \\
\hline 38 & Exhauster intrinsic safety (Off-site) & ECN 626744 \\
\hline 39 & Exhauster PLC & ECN 632390 \\
\hline 40 & Exhauster duct (Off-site) & ECN 628744 \\
\hline 41 & Exhauster heater (Off-site) & $\mathrm{H}-14-100739$ \\
\hline 42 & Exhauster fan and motor assembly (Off-site) & ECN 628744 \\
\hline $43 \pi$ & Inlet breather stack (Off-site) & $\mathrm{H}-14-100742$ \\
\hline 44 & Tank pressure detection (Off-site) & Existing, and $\mathrm{H}+14100521$ \\
\hline 451 & Flammable gas detector (Off-site) & $\mathrm{H}-14-100523$ \\
\hline 46$]$ & X-ray containment (NA) & VI File, Internal memo 75230-96-006 \\
\hline 47 & DS nitrogen purge supply (Off-site) & Existing \\
\hline 48 , & Nitrogen hydrostatic head supply (NA) & Existing \\
\hline $49 \mathrm{~F}$ & Riser sleeve nitrogen purge supply (NA) & H-2-690128, ECN 628706, ECN 62674t \\
\hline 50. & Unique connections (NA) & H-2-690128, ECN 630017 \\
\hline 51) & Truck PLC (Off-site) & ECN 623775 \\
\hline $52 A$ & Audible and visual annunciation (NA) & ECN 623775 \\
\hline 535 & Shutdown interlock (Off-site) & Existing \\
\hline
\end{tabular}




\subsection{DESIGN REVIEW CHECKLIST}

Formal design reviews, as described in WHC-CM-6-1, Standard Engineering Practices, are required to use a design review checklist that has been customized for the review. The checklist developed by the design review committee for this review is listed as an attachment to section 11.0 of this report. The checklist was satisfactorily completed, and all open items from the checklist are discussed in section 10.0 of this report.

\subsection{REVIEW COMMENT RECORDS}

The following table is a listing of all RCR's received during the design review, including an $\mathrm{RCR}$ which was discussed at the final close-out meeting and submitted afterwards. All RCR's were dispositioned and signed off as closed, except for the last RCR, which is discussed as an open item in section 10.0 of this report. A copy of each dispositioned RCR, and the opened RCR, are included as attachments to Section 11.0 of this report.

RCR STATUS 5/7/96 (COB)

\section{Final Design Review, RMCST Modifications for Flammable Gas Tanks}

\begin{tabular}{|c|c|c|}
\hline REVIEWER/DATE/NUMBER & RESP. ENGINEER & $\underline{\text { RCR STATUS }}$ \\
\hline Mousel/0206/1 & Galen Wilson & Withdrawn/Superseded \\
\hline Bogen $/ 0206 / 1$ & Galen Wilson & closed/signed \\
\hline Bogen $/ 0206 / 2$ & Gaten Wi!son & closed/signed \\
\hline Bogen $/ 0206 / 3$ & Galen wilson & closed/signed \\
\hline Bogen/0206/4 & Galen Wilson & closed/signed \\
\hline Bogen/0206/5 & Galen Wilson & closed/signed \\
\hline Bogen $/ 0206 / 6$ & Galen Wilson & closed/signed \\
\hline Bogen/0206/7 & Galen wilson & closed/signed \\
\hline Board/0207/1 & Jim Criddle & closed/signed \\
\hline Board/0207/2 & Jim Criddle & closed/signed \\
\hline Board/0207/3 & Jim Criddle & closed/signed \\
\hline Bogen/0208/1 & Gaten Wilson & closed/signed \\
\hline Bogen/0208/2 & Galen Wilson & closed/signed \\
\hline Bogen/0208/3 & Gelen Hilson & closed/signed \\
\hline Bogen/0208/4 & Gaten wilson & closed/signed \\
\hline Bogen/0208/5 & Galen Wilson & closed/signed \\
\hline Bogen/0208/6 & Galen Wilson & closed/signed \\
\hline White/0214/? & Jim Criddle & Withdrawn/Superseded \\
\hline White $/ 0220 / 1$ & Jim Criddle & closed/signed \\
\hline McElroy $/ 0226 / 1$ & Galen Wilson & closed/signed \\
\hline Martell/0312/1 & Galen wilson & closed/signed \\
\hline Mousel/0312/1 & Galen Wilson & elosed/signed \\
\hline Krogsrud/0312/1 & Galen Wilson & closed/signed \\
\hline
\end{tabular}




\begin{tabular}{|c|c|c|}
\hline REV IEWER/DATE/NUMBER & RESP. ENGINEER & $\underline{\text { RCR STATUS }}$ \\
\hline White/0426/1 & Jim criddle & closed/signed \\
\hline McEl roy $0501 / 1$ & Galen Wilson & closed/signed \\
\hline Jaka/0501/1 & Galen Wilson & closed/signed \\
\hline Tollefson/0504/1 & Galen Hilson & closed/signed \\
\hline Seda/0506/01 & Galen wilson & closed/signed \\
\hline Seda/0506/02 & Galen Wilson & closed/signed \\
\hline Mousel/0507/01 & TBD & OPEN \\
\hline
\end{tabular}

\subsection{CONCLUSIONS AND OPEN ITEMS}

With the approval of this report, the formal design review for the "RMCST Modifications for Flammable Gas Tanks" is completed. The following open items were noted during the design review. These items are scheduled to be completed as part of the normal course of business and are not action items for the design review committee. Therefore, these items are NOT considered to be "open action items" as described in WHC-CM-6-1, EP-4.1, paragraph 2.3.3.2, "Documentation of Action Item Completion."

OPEN ITEM: ATP/OTP testing to demonstrate requirements/operability OPEN ITEM: operation and maintenance procedures revised to reflect modifications

OPEN ITEM: US Bureau of Mines ignitability test report to document sampler and drill string components meet SA requirements for sparking.

The following action items were noted during the design review. These "open action items" will be closed in accordance with the requirements described in WHC-CM-6-1, EP-4.1

ACTION ITEM: Design modification to meet SA requirements related to "safety feature \#8" (pneumatic footclamp); assigned to CED.

ACTION ITEM: Disposition \& closure for open RCR (Shielded Receiver switch); assigned to CED.

The conclusion reached by the design review committee is that the design of the RMCST modifications is acceptable. There are no further action items for the review committee. 


\subsection{DOCUMENTATION}

The following items are provided as attachments to this report:

1. Design Review Checklist

2. Copies of RCR's

3. Meeting Minutes (includes assignments of design review committee members)

Review copies of all ECN's and new drawings provided to committee members for final review are available in the design review file. All released documentation referenced in the report will be available using the controlled document number located in the text where the document is cited. 
WHC-SD-WM-DRR-053, Rev . 0

Page Al-1

ATTACHMENT $\mathbf{1}$

Design Review Checklist 
Documents Reviewed: $\quad$ ECN\# 623775,626740 through 626742,628706 through 628715,628717 , 628720 , \& 630017; H-2 drawing\# 690128, 690131, 690132, 690134, 690136 , \& 690142; WHC-SD-WM-DRR-052, and applicable portions of WHC-SD-WM-SAD-035.

\begin{tabular}{|c|c|c|c|c|c|}
\hline Item & Review Consideration & Yes & No & NA & Remarks \\
\hline 1 & $\begin{array}{l}\text { Have assumptions necessary to perform the design } \\
\text { task been adequately described and are they } \\
\text { reasonable? Where necessary, have assumptions been } \\
\text { identified for reverification when the design task has } \\
\text { been completed? }\end{array}$ & $\mathrm{x}$ & & & Assumptions were conservative. \\
\hline 2 & $\begin{array}{l}\text { Have the appropriate Quality Assurance requirements } \\
\text { been specified? }\end{array}$ & $\mathbf{X}$ & & & \\
\hline 3 & Were sources of information identified? & $\mathrm{x}$ & & & \\
\hline 4 & $\begin{array}{l}\text { Does the design meet the established requirements or } \\
\text { design criteria? }\end{array}$ & $\mathbf{x}$ & & & \\
\hline 5 & $\begin{array}{l}\text { Does the design meet established requirements for } \\
\text { associated system physical and functional interfaces? }\end{array}$ & $\mathbf{x}$ & & & \\
\hline 6 & Are there any interface problems? & $\mathbf{x}$ & & & \\
\hline 7 & $\begin{array}{l}\text { Has appropriste consideration been given to use of } \\
\text { standardized parts, materials and processes, and have } \\
\text { engineering standards and criteria been specified } \\
\text { properly in the design? }\end{array}$ & $\mathrm{x}$ & & & \\
\hline 8 & $\begin{array}{l}\text { Does the design represent the simplest design } \\
\text { consistent with functional requirements and expected } \\
\text { service conditions? }\end{array}$ & & & $\mathrm{X}$ & $\begin{array}{l}\text { Not reviewed, due to schedule } \\
\text { constraints. }\end{array}$ \\
\hline 9 & $\begin{array}{l}\text { Can the equipment be readily assembled/disassembled } \\
\text { as designed? }\end{array}$ & $\mathrm{x}$ & & & \\
\hline 10 & $\begin{array}{l}\text { Does the design minimize overall cost to the extent } \\
\text { practicable? }\end{array}$ & & & $\mathrm{X}$ & $\begin{array}{l}\text { Not reviewed, due to schedule } \\
\text { constraints. }\end{array}$ \\
\hline 11 & $\begin{array}{l}\text { Are the specified materials compatible with each } \\
\text { other and the environmental conditions to which the } \\
\text { material will be exposed? }\end{array}$ & $\mathrm{x}$ & & & \\
\hline 12 & $\begin{array}{l}\text { Are the applicable codes, standards and requirements, } \\
\text { including revisions, properly identified and are their } \\
\text { design requirements provided for? }\end{array}$ & $\mathrm{x}$ & & & \\
\hline 13 & $\begin{array}{l}\text { Have modifications to commercial grade items and } \\
\text { any associated verification operations or tests been } \\
\text { appropriately documented? }\end{array}$ & $\mathbf{x}$ & & & \\
\hline 14 & Have qualified and certified parts been specified? & $\mathrm{x}$ & & & \\
\hline
\end{tabular}




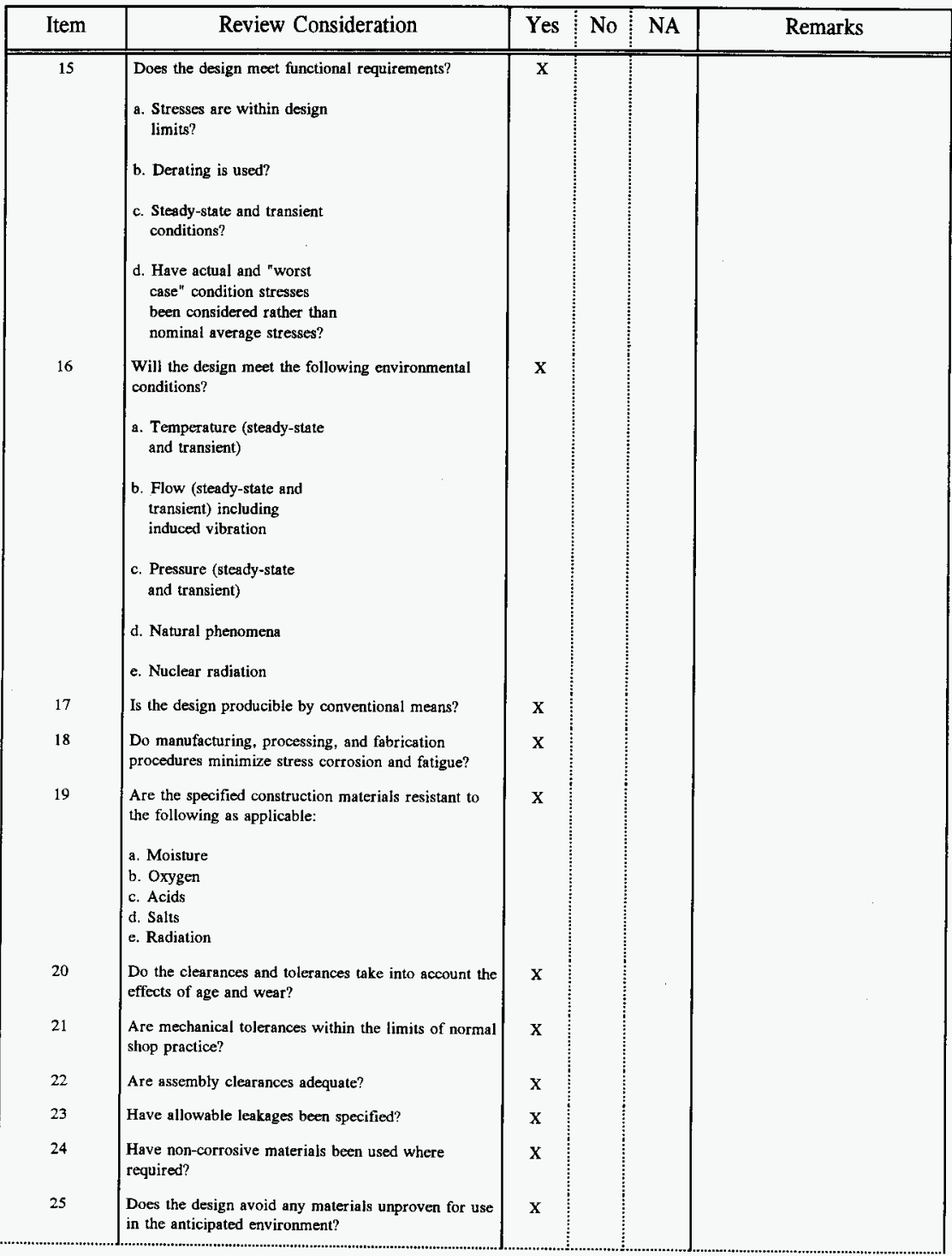




\begin{tabular}{|c|c|c|c|c|c|}
\hline Item & Review Consideration & Yes & No & NA & Remarks \\
\hline 26 & $\begin{array}{l}\text { Can the assembly be stored for extended periods of } \\
\text { time without degrading effects? }\end{array}$ & $\mathbf{X}$ & & & $\begin{array}{l}\text { Applied to equipment required to } \\
\text { enter the tank. }\end{array}$ \\
\hline 27 & $\begin{array}{l}\text { Has the design appropriately considered maintenance, } \\
\text { operation and reliability, including maintensnce } \\
\text { procedures and techniques, unique maintenance } \\
\text { requirements and frequencies? }\end{array}$ & $\mathbf{X}$ & & & \\
\hline 28 & $\begin{array}{l}\text { Are coatings (or finishes) compatible with the } \\
\text { expected environment? With expected usage? }\end{array}$ & $\mathbf{x}$ & & & \\
\hline 29 & $\begin{array}{l}\text { Are surface finish requirements the least stringent } \\
\text { possible? }\end{array}$ & $X$ & & & \\
\hline 30 & $\begin{array}{l}\text { Are required tolerances, fabrication techniques, } \\
\text { processes, etc., consistent with standard practices? }\end{array}$ & $x$ & & & \\
\hline 31 & $\begin{array}{l}\text { Can the design and its parts be easily inspected for } \\
\text { conformance to engineering specifications? }\end{array}$ & $\mathbf{X}$ & & & \\
\hline 32 & $\begin{array}{l}\text { Has adequate accessibility been provided for in- } \\
\text { service inspection? }\end{array}$ & $\mathrm{X}$ & & & \\
\hline 33 & $\begin{array}{l}\text { Does the design meet all established safety } \\
\text { requirements? }\end{array}$ & $\mathrm{x}$ & & & \\
\hline 34 & $\begin{array}{l}\text { Has an acceptable level of radiation exposure been } \\
\text { defined? }\end{array}$ & $X$ & & & \\
\hline 35 & $\begin{array}{l}\text { Have personnel radiation protection requirements } \\
\text { been considered and identified? }\end{array}$ & $x$ & & & \\
\hline 36 & $\begin{array}{l}\text { Have nuclear criticality safety considerations been } \\
\text { incorporated? }\end{array}$ & & & $\mathbf{x}$ & None required. \\
\hline 37 & $\begin{array}{l}\text { Have necessary features been provided to maintain } \\
\text { personnel radiation exposure as low as ressonably } \\
\text { achievable? }\end{array}$ & $\mathbf{x}$ & & & \\
\hline 38 & $\begin{array}{l}\text { Can the hardware be adequately disposed of after use } \\
\text { if it is radiologically or chemically contaminated? }\end{array}$ & $\mathbf{x}$ & & & $\begin{array}{l}\text { Note: The riser sleeve is in } \\
\text { sections. }\end{array}$ \\
\hline 39 & $\begin{array}{l}\text { Have requirements for storing the equipment been } \\
\text { defined? }\end{array}$ & & & $\mathbf{X}$ & Equipment is mobile. \\
\hline 40 & $\begin{array}{l}\text { Have adequate acceptance criteria been specified and } \\
\text { are the verification methods stated appropriately? }\end{array}$ & $\mathrm{x}$ & & & \\
\hline 41 & $\begin{array}{l}\text { Have welding, bolting, joining methods been } \\
\text { adequately specified? }\end{array}$ & $\mathbf{x}$ & & & \\
\hline 42 & Have NDE methods been applied correctly? & $\mathrm{x}$ & & & \\
\hline 43 & $\begin{array}{l}\text { Will a separate Acceptance Test Spec/Procedure be } \\
\text { required? } \\
-\quad \text { If yes, identify responsible organization(s) } \\
\text { for preparation and issue (TBD if unknown) }\end{array}$ & $\mathrm{X}$ & & & $\begin{array}{l}\text { Characterization Equipment } \\
\text { Design will provide ATP's. }\end{array}$ \\
\hline 44 & $\begin{array}{l}\text { Have human factors engineering and operability been } \\
\text { considered? }\end{array}$ & $\mathrm{X}$ & & & \\
\hline 45 & $\begin{array}{l}\text { Is an Operation and Maintenance Manual required? } \\
\text { If so. have requirements been clearly identified? }\end{array}$ & & $\mathbf{x}$ & & $\begin{array}{l}\text { Operating and maintensnce } \\
\text { procedures exist. Characterization } \\
\text { Field Engineering will update. }\end{array}$ \\
\hline
\end{tabular}




\begin{tabular}{|c|c|c|c|c|c|}
\hline Item & Review Consideration & Yes & No & NA & Remarks \\
\hline 46 & $\begin{array}{l}\text { Are current operating documents (procedures, } \\
\text { specifications, etc.) applicable to the design or are } \\
\text { changes necessary? }\end{array}$ & & $\mathrm{X}$ & & $\begin{array}{l}\text { Characterization Field Engineering } \\
\text { will provide documents. }\end{array}$ \\
\hline 47 & $\begin{array}{l}\text { Does the design use engineered safety and operational } \\
\text { protections to avoid an excessive risk-taking } \\
\text { dependence on administrative infallibility? }\end{array}$ & $\mathrm{X}$ & & & \\
\hline 48 & $\begin{array}{l}\text { Are reliability requirements specified? If so, does the } \\
\text { reliability analysis of the design meet the specified } \\
\text { reliability requirements? }\end{array}$ & $\mathbf{x}$ & & & Information is in the SA. \\
\hline 49 & $\begin{array}{l}\text { Have all credible non-standard conditions been } \\
\text { properly considered? }\end{array}$ & $\mathrm{X}$ & & & \\
\hline 50 & $\begin{array}{l}\text { Has the equipment, system, or facility operability } \\
\text { been demonstrated? }\end{array}$ & & $\mathrm{X}$ & & $\begin{array}{l}\text { This will be demonstrated by } \\
\text { OTP. }\end{array}$ \\
\hline 51 & $\begin{array}{l}\text { Is the equipment design adequate to implement the } \\
\text { proposed maintenance philosophy? }\end{array}$ & $\mathbf{X}$ & & & \\
\hline 52 & $\begin{array}{l}\text { If any development work is needed, has it been } \\
\text { funded or performed? }\end{array}$ & $\mathrm{X}$ & & & \\
\hline 53 & Has drawing traceability been provided? & $\mathbf{x}$ & & & \\
\hline 54 & $\begin{array}{l}\text { Has the need for safety analysis of this design been } \\
\text { determined by Safety? }\end{array}$ & $\mathbf{X}$ & & & \\
\hline 55 & $\begin{array}{l}\text { Is the equipment, system, or facility covered by an } \\
\text { existing Safety Analysis Report? If not, complete the } \\
\text { safety analysis in time to incorporate findings of the } \\
\text { analyzed in the design. }\end{array}$ & $\mathbf{X}$ & & & WHC-SD-WM-SAD-035 \\
\hline 56 & $\begin{array}{l}\text { Does the design match the intended (and possible } \\
\text { abnormal) methods of operation of the system or } \\
\text { facility? }\end{array}$ & $\mathbf{X}$ & & & \\
\hline 57 & Is a single point failure analysis required? & $\mathrm{x}$ & & & Completed in the SA. \\
\hline 58 & $\begin{array}{l}\text { Are all indication lights and electrical control } \\
\text { considered fail-safe? }\end{array}$ & $\mathbf{X}$ & & & As required by SA. \\
\hline 59 & $\begin{array}{l}\text { Do the design media, format, content, reproducibility, } \\
\text { and quality comply with all applicable requirements } \\
\text { (including Hanford Plant Standards and referenced } \\
\text { codes and standards)? Are the drawings structured to } \\
\text { meet the needs of users after project completion? }\end{array}$ & $\mathrm{X}$ & & & \\
\hline 60 & $\begin{array}{l}\text { Have availability of power requirements for the } \\
\text { project been verified? }\end{array}$ & $\mathbf{x}$ & & & \\
\hline 61 & $\begin{array}{l}\text { Have requirements for providing as-built drawings } \\
\text { been specified? }\end{array}$ & $\mathrm{x}$ & & & \\
\hline 62 & $\begin{array}{l}\text { Is the design in compliance with applicable regulatory } \\
\text { requirements and/or WHC regulatory commitments? }\end{array}$ & $\mathrm{x}$ & & & \\
\hline 63 & $\begin{array}{l}\text { Are design tolerances appropriate and applied in a } \\
\text { cost-effective manner and are standard materials and } \\
\text { material sizes used where practicable? }\end{array}$ & $\mathbf{x}$ & & & \\
\hline 64 & $\begin{array}{l}\text { Is all computer software and data properly identified } \\
\text { and controlled? }\end{array}$ & $x$ & & & \\
\hline
\end{tabular}


WHC-SD-WM-DRR-053, Rev. 0

Page A2-1

ATTACHMENT 2

Copies of RCR's 


\section{REVIEW COMMENT RECORD (RCR)}

\begin{tabular}{|c|l|}
\hline $\begin{array}{l}\text { 1. Date } \\
02 / 06 / 96\end{array}$ & \multicolumn{2}{|c|}{$\begin{array}{c}\text { 2. Review No. } \\
N / A\end{array}$} \\
\hline $\begin{array}{c}\text { 3. Project No. } \\
\text { N/A }\end{array}$ & 4. Page \\
\end{tabular}

\section{Document Number(s)/Title(s)}

GENERAL
6. Program/Project/ Building Number

Characterization

\section{Reviewer \\ G. J. Bogen}

10. Agreenent with indicated corment disposition(s)

17. Corment Submittal Approval:

Organization Manager (optional

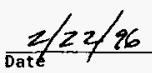

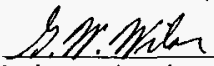

Reviewer/Point of Contact

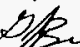

authofiorinator

\section{Organization/Group \\ Characterization Operations}

11. CLOSED
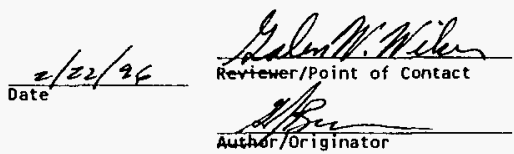

\begin{tabular}{|c|c|c|c|c|}
\hline $\begin{array}{l}12 . \\
\text { Iten }\end{array}$ & $\begin{array}{l}\text { 13. Comment(s)/Discrepancy(s) (Provide technical justification for the } \\
\text { comment and detailed recommendat ion of the action required to correct/ } \\
\text { resolve the discrepancy/problem indicated.) }\end{array}$ & $\begin{array}{l}14 . \\
\text { Hoid } \\
\text { Point }\end{array}$ & 15. Disposition (Provide justification if NOI accepted.) & $\begin{array}{l}16 . \\
\text { status }\end{array}$ \\
\hline 1 & $\begin{array}{l}\text { Designs are to conform to Functional Design Criteria } \\
\text { (FDC) referencing unreleased Safety Assessment (SA)? } \\
\text { what controls have been establ ished to ensure } \\
\text { designs will conform to "final" (released) SA? }\end{array}$ & & $\begin{array}{l}\text { A Compliance Matrix will be sent to review } \\
\text { board members when Safety Assessment is in } \\
\text { final draft. }\end{array}$ & \\
\hline 2 & $\begin{array}{l}\text { Review not inclusive of all required changes, i.e.: } \\
\text { Operating Procedure, PM's, Set Points, Required } \\
\text { Testing (envelope, sparking), Sampler Design, } \\
\text { Training, Interlocks, Quill Rod, SDD/Interface }\end{array}$ & & $\begin{array}{l}\text { Out of Scope of this review these items to } \\
\text { be addressed by Readiness Review. }\end{array}$ & \\
\hline & & & & \\
\hline & & & & \\
\hline & & & & \\
\hline & & & & \\
\hline & & & & \\
\hline & & & & \\
\hline & & & & \\
\hline & & & & \\
\hline & & & & \\
\hline & & & & \\
\hline
\end{tabular}




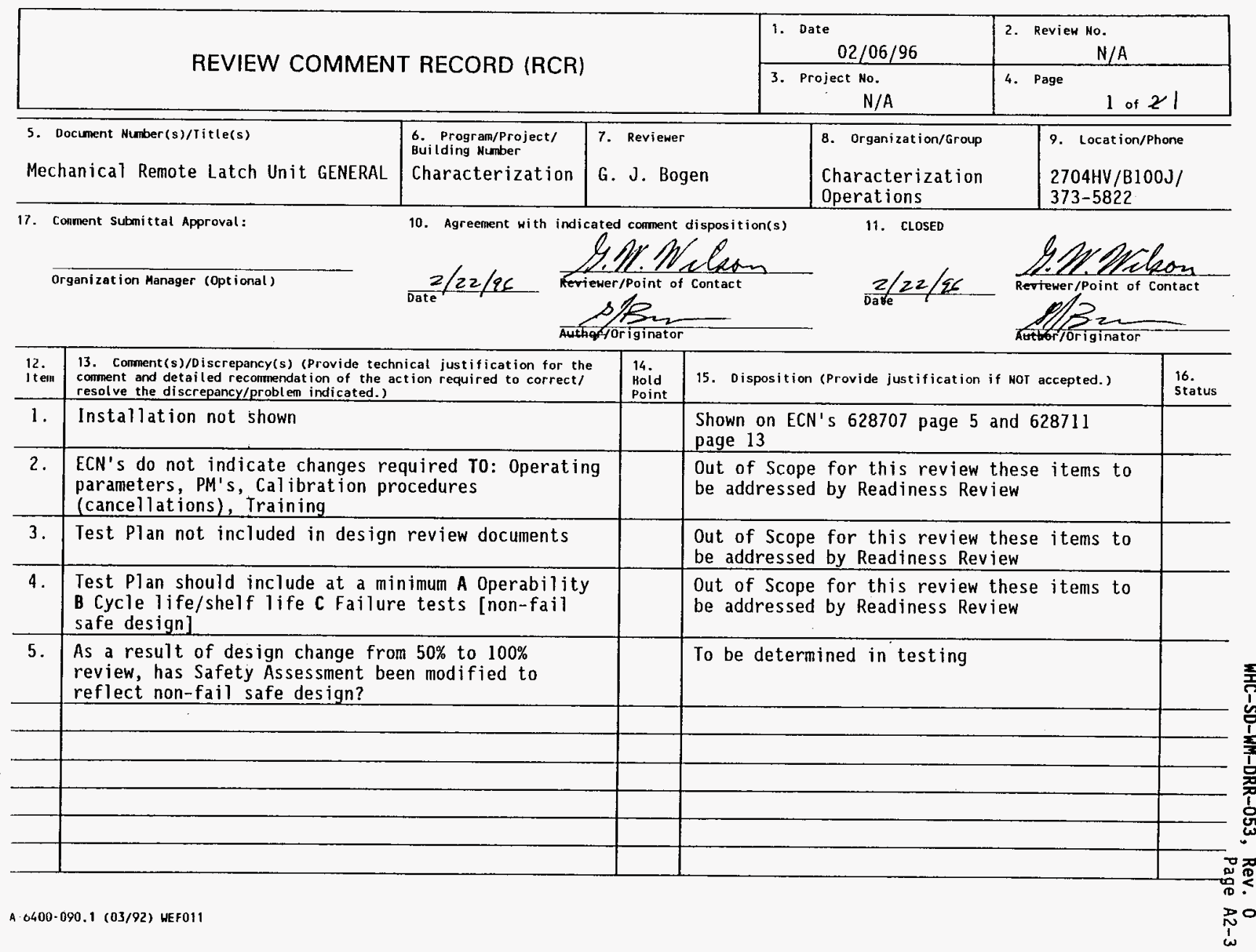




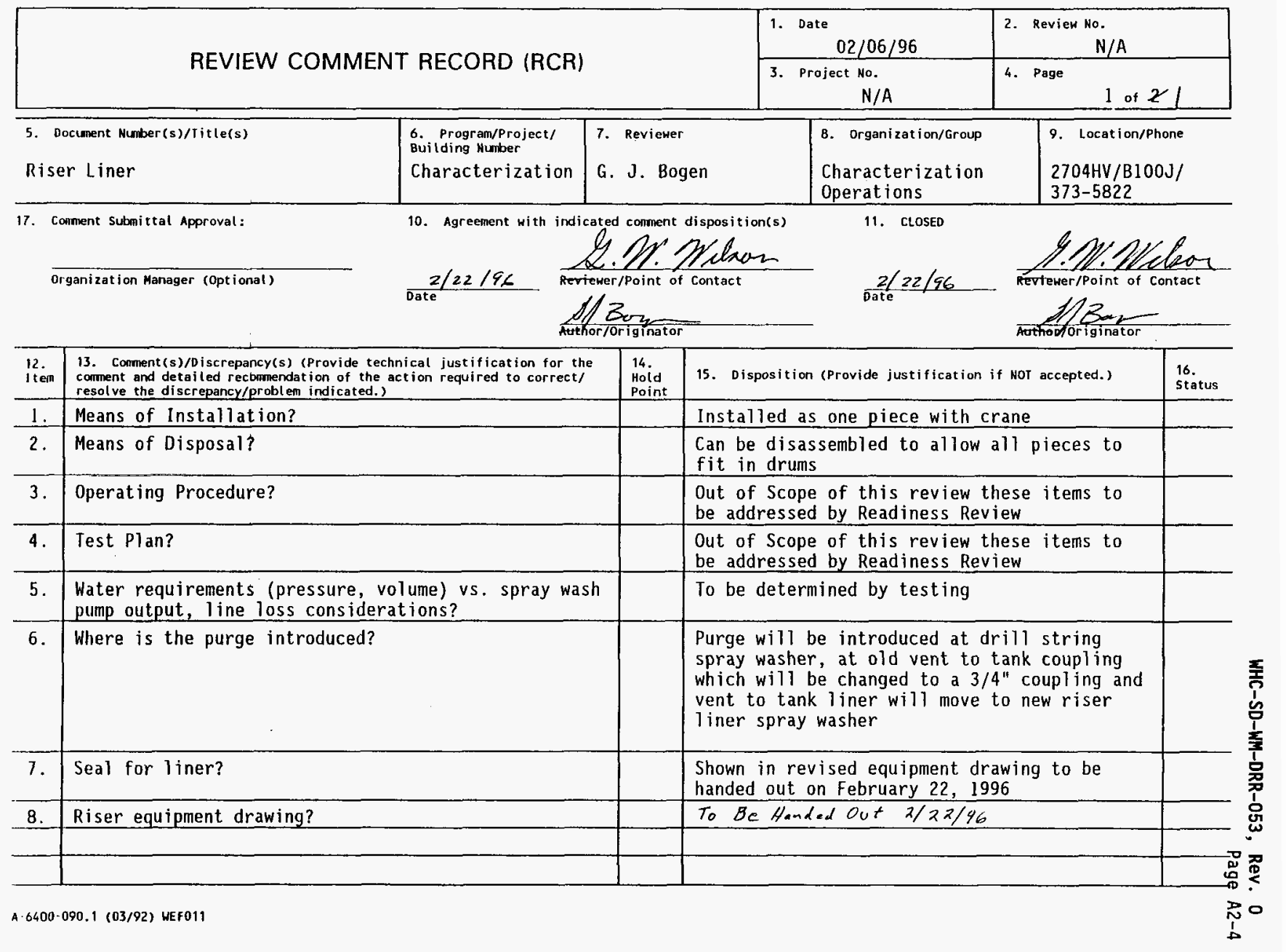




\begin{tabular}{|l|l|l|l|}
\hline REVIEW COMMENT RECORD (RCR) & $\begin{array}{l}\text { Date Review No. } \\
\text { N/A }\end{array}$ & $\begin{array}{l}02 / 06 / 96 \\
3 . \text { Project No. N/A } \\
\text { N/A }\end{array}$ & $\begin{array}{l}4 . \text { Page } \\
1 \text { of } 21\end{array}$ \\
\hline
\end{tabular}

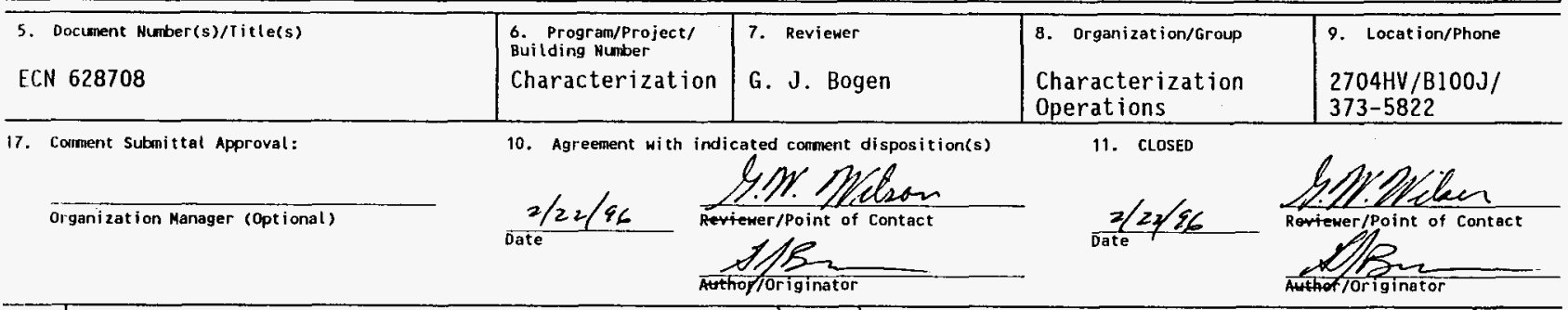

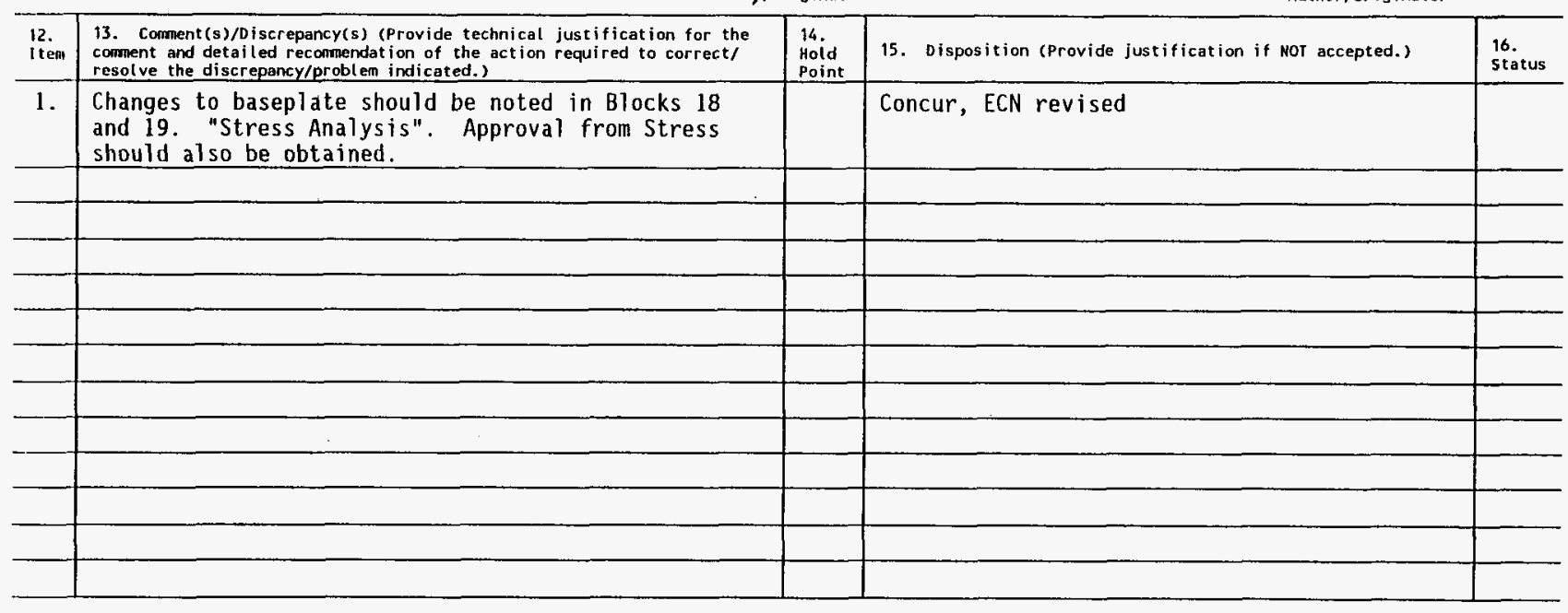




\section{REVIEW COMMENT RECORD (RCR)}

\begin{tabular}{|c|c|}
\hline $\begin{array}{l}\text { 1. Date } \\
02 / 06 / 96\end{array}$ & \multicolumn{2}{|c|}{ Review No. } \\
\hline $\begin{array}{c}\text { 3. Project No. } \\
\text { N/A }\end{array}$ & 4. Page \\
\hline
\end{tabular}

5. Document Mumber(s)/Title(s)

\section{ECN 628709}

17. Connent Submittal Approval:

Organization Manager (Optional)

\begin{tabular}{l|ll}
$\begin{array}{l}\text { 6. Program/Project/ } \\
\text { Building Nuber } \\
\text { Characterization }\end{array}$ & 7. Reviewer \\
\end{tabular}

10. Agreement with indicated comment disposition(s)

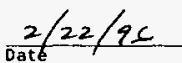

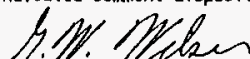

Roviower/point of contact
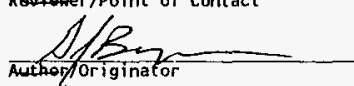

8. Organization/Group

Characterization Operations
9. Location/Phone

2704HV/B100J/ $373-5822$

\section{CLOSED}
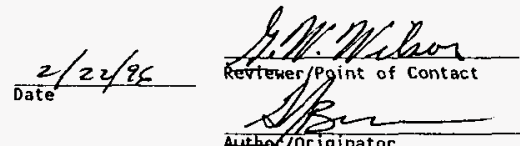

Autheriorionator

\begin{tabular}{|c|c|c|c|c|}
\hline $\begin{array}{l}12 . \\
1 \text { tem }\end{array}$ & $\begin{array}{l}\text { 13. Conment(s)/Discrepancy(s) (Provide technical justification for the } \\
\text { comnent and detailed recommendation of the action required to correct/ } \\
\text { resolve the discrepancy/problem indicated.) }\end{array}$ & $\begin{array}{l}\text { Thi } \\
\text { Hoid } \\
\text { Point }\end{array}$ & 15. Disposition (Provide justification if NOT accepted.) & $\begin{array}{l}16 . \\
\text { Status }\end{array}$ \\
\hline 1. & Sheet 5 and 6 should be switched & & Concur, ECN revised & \\
\hline 2. & $\begin{array}{l}\text { Item } 88 \text { is a pressure barrier. Stress calculations } \\
\text { or approval from Stress SB included on ECN }\end{array}$ & & Concur, ECN revised & \\
\hline 3. & Bolt torque SB specified & & Concur, ECN revised & \\
\hline & & & & \\
\hline & & & & \\
\hline & & & & \\
\hline & & & & \\
\hline & & & & \\
\hline & & & & \\
\hline & & & & \\
\hline & & & & \\
\hline & & & & \\
\hline & & & & \\
\hline & & & & \\
\hline & & & & \\
\hline
\end{tabular}




\section{REVIEW COMMENT RECORD (RCR)}

\begin{tabular}{|c|l|}
\hline \begin{tabular}{|l|} 
1. Date \\
$02 / 06 / 96$
\end{tabular} & \multicolumn{1}{|c|}{ 2. Revien No. } \\
\hline $\begin{array}{c}\text { 3. Project No. } \\
\text { N/A }\end{array}$ & 4. Page \\
\hline
\end{tabular}

\section{Document Number(s)/Title(s)}

\section{ECN 628713}

17. Comment Subnittal Approval:

\begin{tabular}{l|l}
$\begin{array}{l}\text { 6. Progran/Project/ } \\
\text { Building Number } \\
\text { Characterization }\end{array}$ & 7. Reviewer \\
& G. J. Bogen
\end{tabular}

10. Agreement with indicated comment disposition(s)

Organi zat ion Manager (Optional)
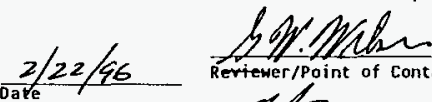

Reriewer/Point of Contact

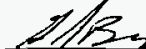

Authof/originator
8. Organization/Group

Characterization Operations

11. CLOSED

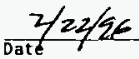

9. Location/Phone

$2704 \mathrm{HV} / \mathrm{B} 100 \mathrm{~J} /$ 373-5822

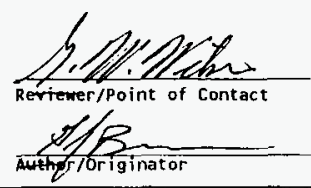

\begin{tabular}{|c|c|c|c|c|}
\hline 12. & $\begin{array}{l}\text { 13. Conment(s)/Discrepancy(s) (Provide technical justification for the } \\
\text { comment and detailed recomendation of the action required to correct/ } \\
\text { resolve the discrepancy/problem indicated.) }\end{array}$ & $\begin{array}{l}14 . \\
\text { Hoid } \\
\text { Point }\end{array}$ & 15. Disposition (Provide justification if NOT accepted.) & $\begin{array}{l}16 . \\
\text { Status }\end{array}$ \\
\hline 1. & ECN Block 13b. Description is wrong. & & Concur, ECN revised & \\
\hline 2. & Is weather cover going to be pressure/leak tested? & & No, normal pressure range is 5-10" water & \\
\hline & & & & \\
\hline & & & & \\
\hline & & & & \\
\hline & & & & \\
\hline & & & & \\
\hline & & & & \\
\hline & & & & \\
\hline & & & & \\
\hline & & & & \\
\hline & & & & \\
\hline & & & & \\
\hline & & & & \\
\hline
\end{tabular}




\section{REVIEW COMMENT RECORD (RCR)}

\begin{tabular}{|c|c|}
\hline $\begin{array}{l}\text { 1. Date } \\
02 / 06 / 96\end{array}$ & $\begin{array}{r}\text { 2. Review No. } \\
N / A\end{array}$ \\
\hline $\begin{array}{l}\text { 3. Project No. } \\
N / A\end{array}$ & 4. Page \\
\hline
\end{tabular}

\section{Document Number(s)/Titte(s)}

ECN 628717

17. Comnent Submittal Approval:

Organization Manager (Optional)

\begin{tabular}{l|l}
$\begin{array}{l}\text { 6. Program/Project/ } \\
\text { Building Number }\end{array}$ & 7. Reviewer \\
Characterization & G. J. Bogen \\
\hline
\end{tabular}

10. Agreement with indicated coment disposition(s) $2 / 22 / 96$ Reviever/Point of contact

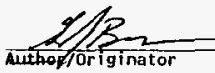

8. Organization/Group

Characterization

Operations

11. CLOSED

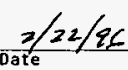

$2704 \mathrm{HV} / \mathrm{B} 100 \mathrm{~J} /$

$373-5822$

9. Location/Phone

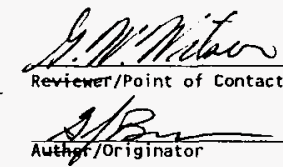

ginator

\begin{tabular}{|c|c|c|c|c|}
\hline $\begin{array}{l}12 . \\
\text { Iten }\end{array}$ & $\begin{array}{l}\text { 13. Comment(s)/Discrepancy(s) (Provide technical justification for the } \\
\text { comment and detailed recommendation of the action required to correct/ } \\
\text { resolve the discrepancy/problem indicated.) }\end{array}$ & $\begin{array}{l}14 . \\
\text { Hoid } \\
\text { Point }\end{array}$ & 15. Oisposition (Provide justification if NOT accepted.) & $\begin{array}{l}16 . \\
\text { Status }\end{array}$ \\
\hline 1. & Pages numbered improperly & & Concur, ECN revised & \\
\hline 3. & ECN poorly identifies where changes have occurred & & $\begin{array}{l}\text { Would create more problems by "clouding" } \\
\text { changes }\end{array}$ & \\
\hline & & & & \\
\hline & & & & \\
\hline & & & & \\
\hline & & & & \\
\hline & & & & \\
\hline & & & & \\
\hline & & & & \\
\hline & , & & & \\
\hline 6400 & 990.1 (03/92) WEF011 & & & \\
\hline
\end{tabular}




\section{REVIEW COMMENT RECORD (RCR)}

\begin{tabular}{|l|l|}
\hline $\begin{array}{l}\text { 1. } \begin{array}{l}\text { Date } \\
\text { February 7, 1996 }\end{array} \\
\text { 3. Project No. } \\
\text { CHARACTERIZATION }\end{array}$ & $\begin{array}{l}\text { 2. Review No. } \\
6020703\end{array}$ \\
\hline
\end{tabular}

\section{Document Number(s)/Title(s)}

RMCST $3 \& 4$ MOOIFICATION ECN \#626742

17. Coment Submittal Approval:

Orgenization Menager (optional)

Orgenization Manager (optional)

\begin{tabular}{l|l}
$\begin{array}{l}\text { 6. Program/Project/ } \\
\text { Building Mumber } \\
\text { CHARACTERIZATION }\end{array}$ & 7. Reviewer \\
$\begin{array}{l}\text { J. CORBETT } \\
\text { D. BOARD }\end{array}$
\end{tabular}

10. Agreement with jodicated comment disposition(s)

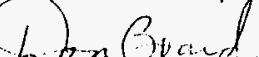

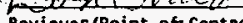

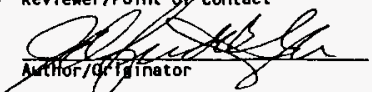

8. Organization/Group

CHARACTERIZATION QUALITY ASSURANCE

11. CLOSED
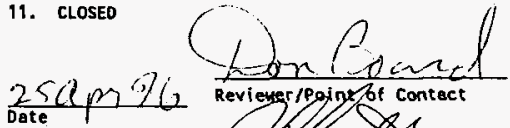

2704HV/F2OOH 373-5191

Reviester/egint/of Contect

12. 13. Coment(s)/Discrepancy(s) (Provide technical justification for the comment and detailed recommendation of the action required to correct/ resolve the discrepaney/problem indicoted, )

1. Page 4 - On H-2-690071, Sht 4, Rev 1, Recommend the drawing terminal block be labeled TB5 in the Control Console.

2. Page 4 - On H-2-690071, Sht 4, Rev 1, The deletions do not appear to have been made on the drawing.

3. Page 5 - On H-2-690071, Sht 6, Rev 1, Drawing changes do not appear to have been made for:

Line 2 - REMOVE the were connected to the left Jl-d

Line 3 - REMOVE the wire connected to the left Jl-e

Line 4 - DELETE the text to the left of the 2 wires Line 15 - For All wires connected to the left of P2

14.

Hold

15. Disposition (Provide justification if NOT accepted.)

Accept, correct label is CC-TB5.

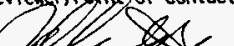

suthor ofiginetor
Accept, ECN changes will be incorporated on drawing.

Accept, ECN changes will be incorporated on drawing.

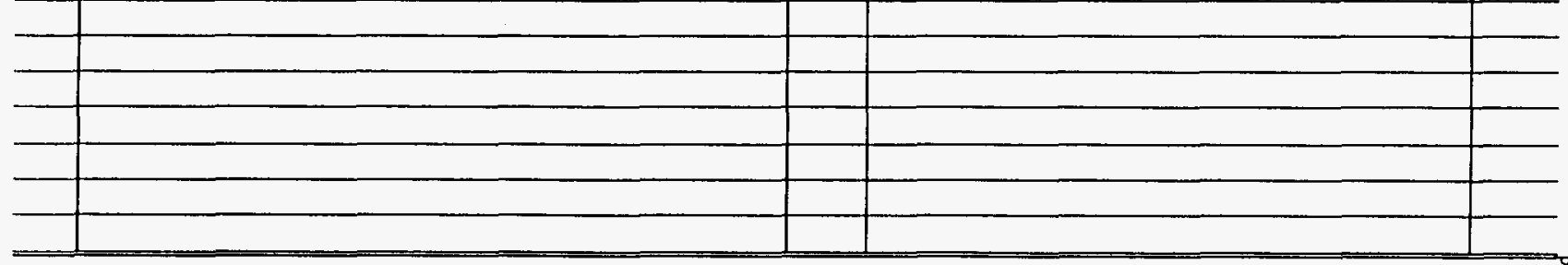




\section{REVIEW COMMENT RECORD (RCR)}

1. Date

February 7,1997

3. Project Mo. CHARACTERIZATION
2. Review No. 6020702

4. Page

1 of 1
5. Document Nuber(s)/Title(s)

RMCST $3 \& 4$

ECN \#626741

17. Comment Submittal Approval:

Orgenization Menager (Optional)

\begin{tabular}{l|l}
$\begin{array}{l}\text { 6. Program/Project/ } \\
\text { Building Number }\end{array}$ & 7. Reviewer \\
CHARACTERIZATION & D. BOARD
\end{tabular}

J. CORBETT

10. Agreement with indicated comment disposition(s)<smiles>CC(C)C1CCCCC1C1CCCCC1</smiles>

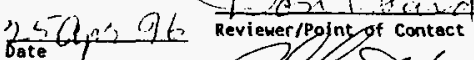

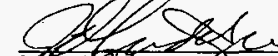

8. Organi zation/Group
CHARACTERIZATION
QUALITY ASSURANCE

9. Location/Phone

2704HV/F20OH

$373-5191$

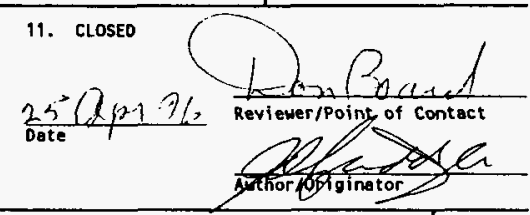

12. 13. Comment(s)/Discrepancy(s) (Provide technical justiflcation for the I tem coment and detailed recommendation of the action required to correct/ resolve the discrepancy/problem indicated.)

1. Page 3, H-2-690068, sht 6, rev. 1, The drawing changes for the $p l a t e s$ " $S$ " and "G" have been interchanged.

2. Page 3, H-2-690073, sht. I, rev. 1, The drawing description and material designation of part \#41 has not been corrected.

\begin{tabular}{|c|l|l}
\hline $\begin{array}{c}\text { 14.id } \\
\text { koid } \\
\text { Point }\end{array}$ & 15. Disposition (Provide justification if Nor accepted.) & $\begin{array}{l}16 . \\
\text { stotus }\end{array}$ \\
\hline DB & Accepted, corrections will be made. & \\
\hline DB & Accepted, corrections will be made. & \\
\hline & & \\
\hline & & \\
\hline & & \\
\hline & & \\
\hline & & \\
\hline & & \\
\hline
\end{tabular}




\section{REVIEW COMMENT RECORD (RCR)}

1. Dete
February 7, 1996
3. Project No. CHARACTERIZATION
2. Review Ho. $6020701 \mathrm{C}$

4. Page 1 of 1

\section{Document Munber(s)/Title(s)}

\section{RMCST $3 \& 4$ MODIFICATION} ECN $\# 626740$

17. Comment Submittal Approval:

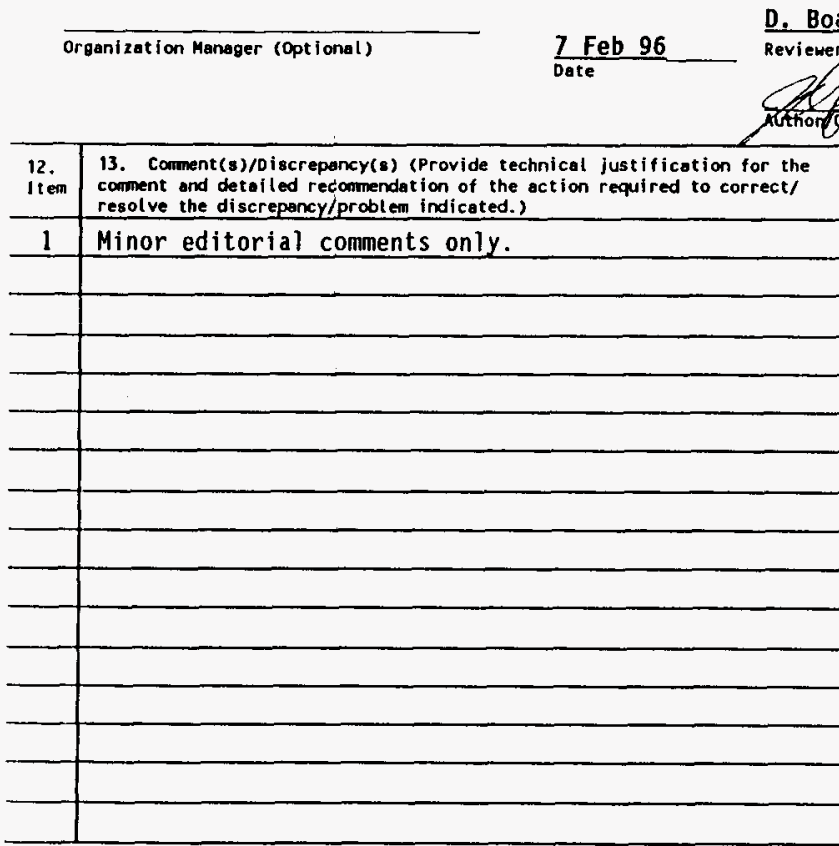

\begin{tabular}{l|l}
$\begin{array}{l}\text { 6. Program/Project/ } \\
\text { Buil lding Humber } \\
\text { CHARACTERIZATION }\end{array}$ & 7. Reviewer \\
D. BOARD
\end{tabular}
J. CORBETT

10. Agreement with indicated comment disposition(s)

\section{Board} pojnt of contact

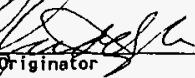

\begin{tabular}{|l|l|}
\hline 8. Organization/Group & 9. Location/Phone \\
CHARACTERIZATION & $2704 \mathrm{HV} / \mathrm{F} 200 \mathrm{H}$ \\
QUALITY ASSURANCE & $373-5191$ \\
\hline
\end{tabular}

11. CLOSED

\section{Feb 96} Date

D. Board Reviewer/Poing Contoct 14.

\begin{tabular}{|l|l|c}
\hline $\begin{array}{l}14 . \\
\text { Hoid } \\
\text { Point }\end{array}$ & 15. Disposition (Provide justification if MOT accepted.) & $\begin{array}{l}\text { 16. } \\
\text { Status }\end{array}$ \\
\hline & None required & C \\
\hline & & \\
\hline & & \\
\hline & & \\
\hline & & \\
\hline & & \\
\hline & & \\
\hline & & \\
\hline & & \\
\hline
\end{tabular}




\section{REVIEW COMMENT RECORD (RCR)}

1. Date $02 / 08 / 96$

3. Project No.

N/A
2. Review No.

N/A)

4. Page

1 of 21
5. Document Number(s)/Title(s)

Mechanica] RLU - General

17. Corment Submittal Approval:

Organization Manager (optional)

\begin{tabular}{l|l}
$\begin{array}{l}\text { 6. Program/Project/ } \\
\text { Building Number } \\
\text { Characterization }\end{array}$ & 7. Reviewer \\
& G. J. Bogen
\end{tabular}

10. Agreement with indicated conment disposition(s)

$2 / 22 / 26$
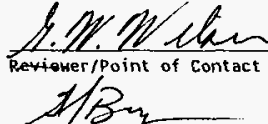

Autrof/Originato

\section{Organization/Group \\ Characterization \\ Operations}

11. CLOSED
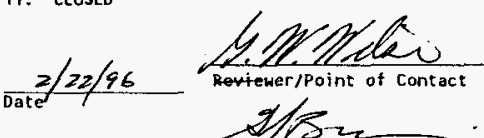

Awtheroriginato

9. Location/Phone

$2704 \mathrm{HV} / \mathrm{B} 100 \mathrm{~J} /$ $373-5822$

ate $27 / 96$

act

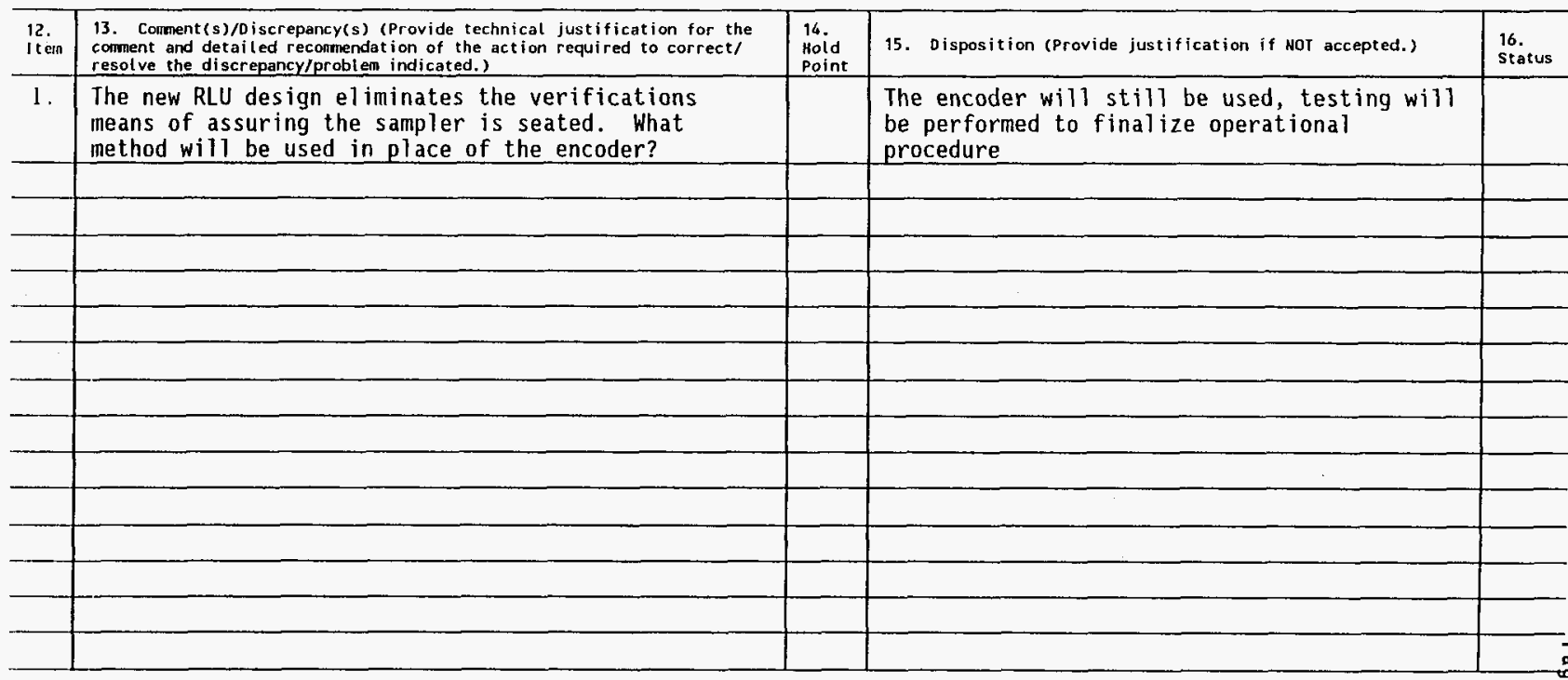

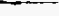

tus 


\section{REVIEW COMMENT RECORD (RCR)}

\begin{tabular}{|c|c|}
\hline $\begin{array}{l}\text { 1. Date } \\
02 / 08 / 96\end{array}$ & $\begin{array}{r}\text { 2. Review No. } \\
\text { N/A }\end{array}$ \\
\hline $\begin{array}{r}\text { 3. Project No. } \\
\text { N/A }\end{array}$ & 4. Page \\
\hline
\end{tabular}

\section{Document Number(s)/Title(s)}

\section{ECN 628706}

\begin{tabular}{l|l}
$\begin{array}{l}\text { 6. Program/project/ } \\
\text { Building Number } \\
\text { Characterization }\end{array}$ & 7. Reviewer \\
& G. J. Bogen \\
\hline
\end{tabular}

10. Agreenent with indicated coment disposition(s)

Organization Manager (optional)

\section{B. Organization/Group \\ Characterization Operations}

11. CLOSED

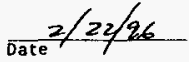

9. Location/Phone

$2704 \mathrm{HV} / \mathrm{Bl}$ 100J/ 373-5822
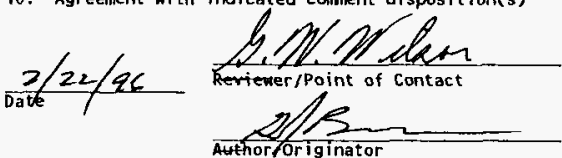

17. Comment Submittal Approval (1)

\begin{tabular}{|c|c|c|c|c|}
\hline \multicolumn{3}{|c|}{ Authorforiginator } & \multicolumn{2}{|c|}{ Atthr/originator } \\
\hline $\begin{array}{l}12 . \\
1 \text { tern }\end{array}$ & $\begin{array}{l}\text { 13. Comment(s)/Discrepancy(s) (Provide technical justification for the } \\
\text { comment and detailed recommendation of the action required to correct/ } \\
\text { resolve the discrepancy/problem indicated.) }\end{array}$ & $\begin{array}{l}14 . \\
\text { Hoid } \\
\text { Point }\end{array}$ & 15. Disposition (Provide justification if Nor accepted.) & $\begin{array}{l}16 . \\
\text { Status }\end{array}$ \\
\hline 1. & $\begin{array}{l}\text { Sheet 4. Part Number } 68 \text { "ASTM A36" is correct call- } \\
\text { out. }\end{array}$ & & Concur, ECN revised & \\
\hline 2. & $\begin{array}{l}\text { Sheets } 4 \text { and } 5 \text {. Part number } 56 \text { and note } 20 \text { do not } \\
\text { agree in units (GPM, SCFM). }\end{array}$ & & Concur, ECN revised & \\
\hline 3. & $\begin{array}{l}\text { Sheets } 8 \text { and } 9 . \text { Inadequate dimensioning of part } \\
\text { number } 68 . \text { (Sheet } 8 ; \text { hidden line on left side). }\end{array}$ & & Concur, ECN revised & \\
\hline 4. & $\begin{array}{l}\text { Sheet } 21 \text {. Dimensions on left and right of top view } \\
\text { are illegible }\end{array}$ & & Concur, ECN revised & \\
\hline & & & & \\
\hline & & & & \\
\hline & & & & \\
\hline & & & & \\
\hline & & & & \\
\hline & & & & \\
\hline & & & & \\
\hline & & & & \\
\hline
\end{tabular}

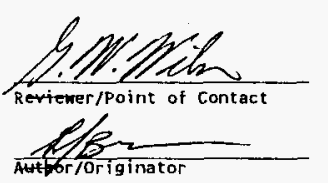




\begin{tabular}{|c|l|l|l|}
\hline \multirow{2}{*}{ REVIEW COMMENT RECORD (RCR) } & $\begin{array}{l}\text { 1. Date Review No. } \\
\text { N/A }\end{array}$ \\
\cline { 2 - 3 } & $\begin{array}{c}3 . \text { Project No. } \\
\text { N/A }\end{array}$ \\
\hline
\end{tabular}

\begin{tabular}{l|l|l|l|l|l|l}
\hline 5. Document Number(s)/Iitle(s) & $\begin{array}{l}\text { 6. Program/Project/ } \\
\text { Building Nuber } \\
\text { Characterization }\end{array}$ & $\begin{array}{l}\text { 7. Reviewer } \\
\text { G. J. Bogen } 628706\end{array}$ & $\begin{array}{l}\text { 8. Organization/Group } \\
\text { Characterization } \\
\text { Operations }\end{array}$ & $\begin{array}{l}\text { 9. Location/Phone } \\
2704 H V / B 100 J / \\
373-5822\end{array}$ \\
\hline
\end{tabular}

17. Comment Submittal Approval:

Organization Manager (Optional)
10. Agreement with indicated comment disposition(s) $22 / 22 / 96$

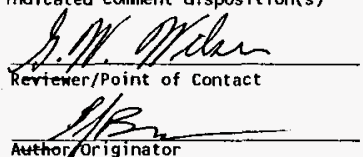

11. CLOSED

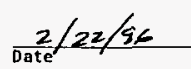

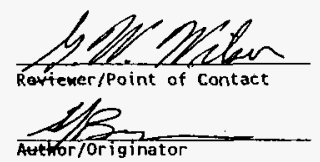

Authr/originator

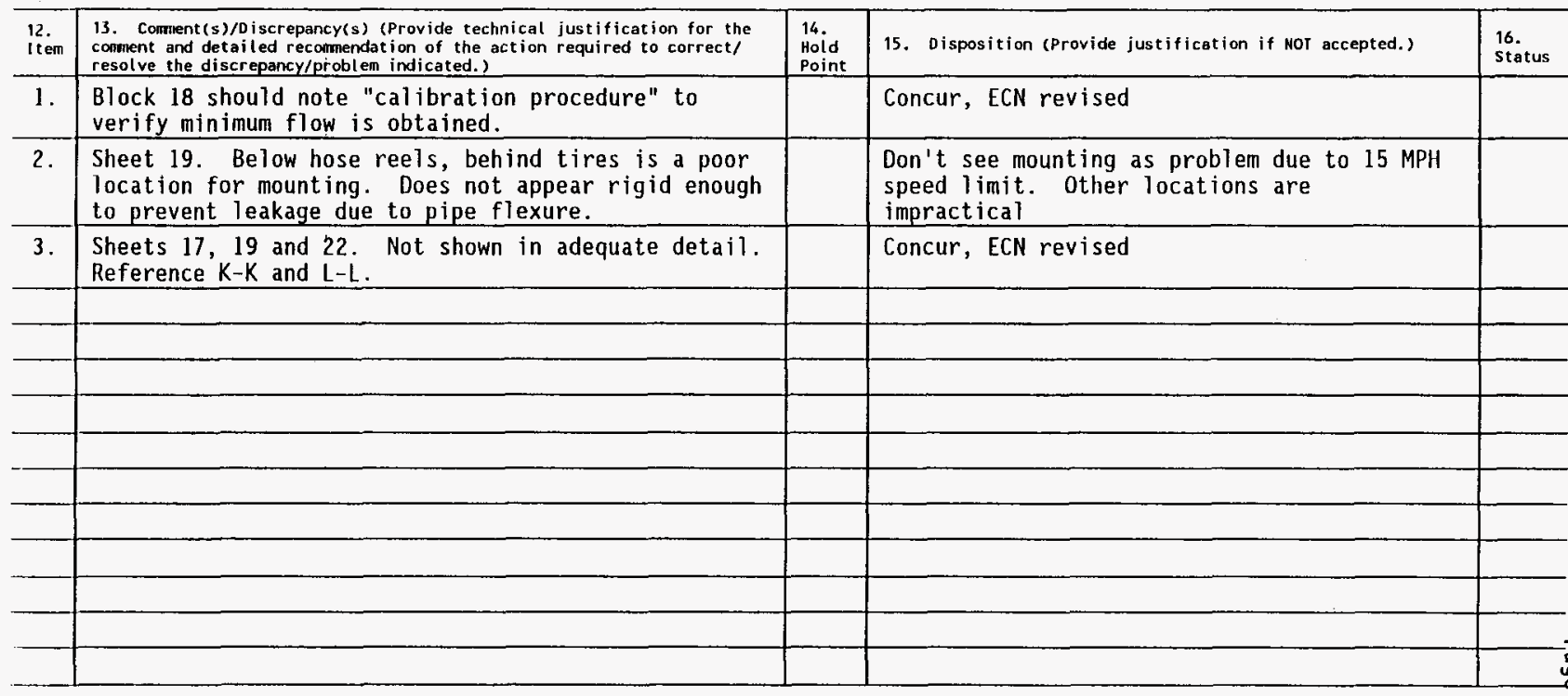




\section{REVIEW COMMENT RECORD (RCR)}

1. Date
$02 / 08 / 96$

3. Project No.
2. Revieh No.

$N / A$

4. Page

1 of 21
5. Document Number(s)/Title(s)

\section{ECN 628707}

17. Comment submittal Approval:

Organization Manager (Optional)

\begin{tabular}{l|l}
$\begin{array}{l}\text { 6. Program/Project/ } \\
\text { Building Number } \\
\text { Characterization }\end{array}$ & 7. Reviewer \\
& G. J. Bogen
\end{tabular}

10. Agreement with indicated connent disposition(s)

$\frac{2}{\text { Date }} / 22 / 26$
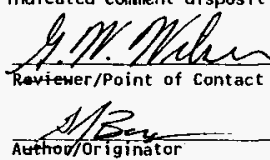

8. Organization/Group

Characterization

Operations

11. CLosED

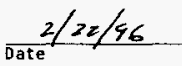

9. Location/Phone

2704HV/B100J/ 373-5822

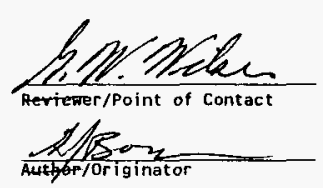

\begin{tabular}{|c|c|c|c|c|}
\hline item & $\begin{array}{l}\text { 13. Comment(s)/Discrepancy(s) (Provide technical justification for the } \\
\text { comment and detailed recommendation of the action required to correct/ } \\
\text { resolve the discrepancy/problem indicated.) }\end{array}$ & $\begin{array}{l}14 . \\
\text { Hold } \\
\text { Point }\end{array}$ & 15. Disposition (Provide justification if NOT accepted.) & $\begin{array}{l}16 . \\
\text { status }\end{array}$ \\
\hline 1. & $\begin{array}{l}\text { Sheets } 5 \text { and } 6 \text {. Changes in addition to those } \\
\text { identified on sheet } 5 \text { have been made. [or appear to } \\
\text { have been made]. }\end{array}$ & & $\begin{array}{l}\text { Weather cover changes are made per other } \\
\text { released ECN's }\end{array}$ & \\
\hline & & & & \\
\hline & & & & . \\
\hline & & & & \\
\hline & & & & \\
\hline & & & & \\
\hline & & & & \\
\hline & & & & \\
\hline & & & & \\
\hline & & & & \\
\hline & & & & \\
\hline
\end{tabular}




\section{REVIEW COMMENT RECORD (RCR)}

\begin{tabular}{|l|l|}
\hline \begin{tabular}{|l|} 
1. Date \\
$02 / 08 / 96$
\end{tabular} & \multicolumn{2}{|c|}{$\mathrm{N} / \mathrm{A}$} \\
\hline $\begin{array}{c}\text { 3. Project No. } \\
\mathrm{N} / \mathrm{A}\end{array}$ & 4. Page \\
\hline
\end{tabular}

5. Document Nunber(s)/Iitle(s)

ECN 628709

17. Comment Submittal Approval

organi zation Manager copt ional
6. Progran/Project/ Building Number

Characterization

\section{Reviewer \\ G. J. Bogen}

10. Agreement with indicated comment disposition(s)

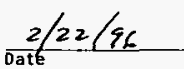

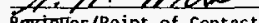

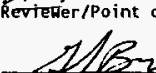

8. Organization/Group

Characterization

Operations

11. CLOSED

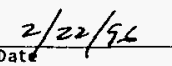

9. Location/Phone

$2704 \mathrm{HV} / \mathrm{B} 10 \mathrm{OJ}$

373-5822

12. 13. Comment(s)/Discrepancy(s) (Provide technical justification for the

It ein comment and detailed recommendation of the action required to correct/ resolve the discrepancy/problem indicated.)

1. Sheets 3,7 and 10 (two places) part number 86 should be changed to part number 88 (total 4 places)

\section{4.} Hold Point

15. Disposition (Provide justification if NOT accepted.)

Concur, ECN revised

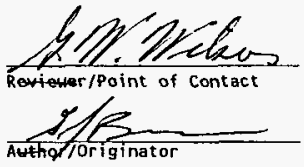
Status

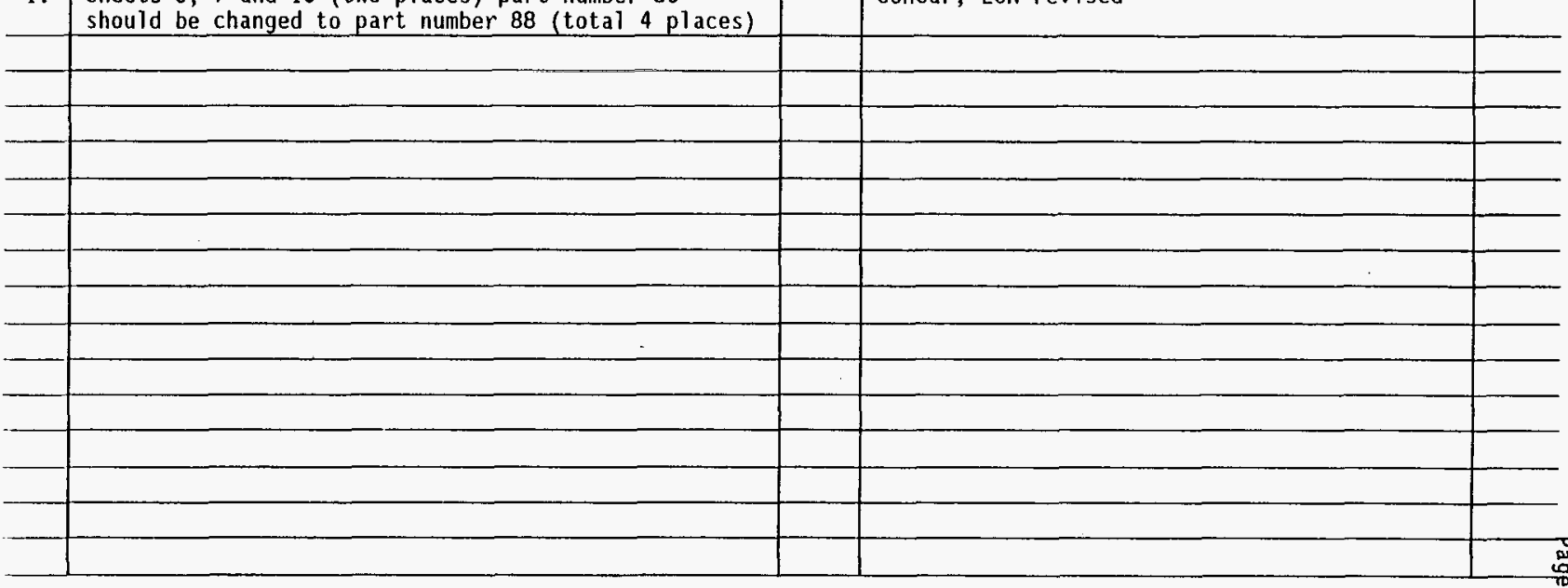




\begin{tabular}{|c|l|l|l|}
\hline \multirow{2}{*}{ REVIEW COMMENT RECORD (RCR) } & $\begin{array}{l}\text { 1. Date } \\
02 / 08 / 96\end{array}$ & $\begin{array}{l}\text { Review No. } \\
\text { N/A }\end{array}$ \\
\cline { 2 - 4 } & $\begin{array}{l}3 . \text { Project No. } \\
\text { N/A }\end{array}$ & $\begin{array}{l}4 . \text { Page } \\
1\end{array}$ \\
\hline
\end{tabular}

5. Document Number(s)/Jitle(s)

\section{ECN 628712}

17. Comnent Submittal Approval:

Organization Manager (Optional)

\begin{tabular}{l|ll}
$\begin{array}{l}\text { 6. Program/Project/ } \\
\text { Building Number } \\
\text { Characterization }\end{array}$ & 7. Reviewer \\
& G. J. Bogen
\end{tabular}

Building Number

30. Agreement with indicated comment disposition(s)

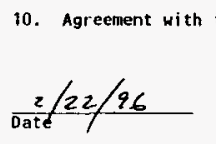

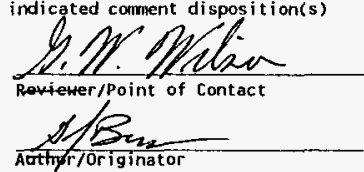

8. Organization/Group

Characterization

Operations

11. CLOSEO

$\frac{2}{\text { Dat6 }} 22 / 96$
9. Location/Phone

2704HV/B100J/

373-5822

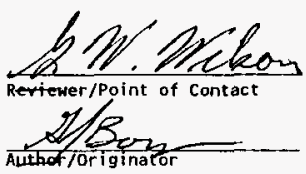

\begin{tabular}{|c|c|c|c|c|}
\hline \begin{tabular}{l|l|l}
2. \\
Item
\end{tabular} & $\begin{array}{l}\text { 13. Comment(s)/Discrepancy(s) (Provide technical justification for the } \\
\text { comment and detailed recommendat ion of the action required to correct// } \\
\text { resolve the discrepancy/problem indicated.) }\end{array}$ & $\begin{array}{l}14 . \\
\text { Hoid } \\
\text { Point }\end{array}$ & 15. Disposition (Provide justification if NOT accepted.) & $\begin{array}{l}16 . \\
\text { status }\end{array}$ \\
\hline 1. & Sheet 3 "zinc" is misspelled. & & Concur, ECN revised & \\
\hline 2. & Sheets 4 and 6. Item 17 is not installed. & & Concur, reference note from sheet 6 & \\
\hline & & & & \\
\hline & & & & \\
\hline & & & & \\
\hline & & & & \\
\hline & & & & \\
\hline & & & & \\
\hline & & & & \\
\hline & & & & \\
\hline & & & & \\
\hline & & & & \\
\hline & & & & \\
\hline & & & & \\
\hline & & & & \\
\hline
\end{tabular}




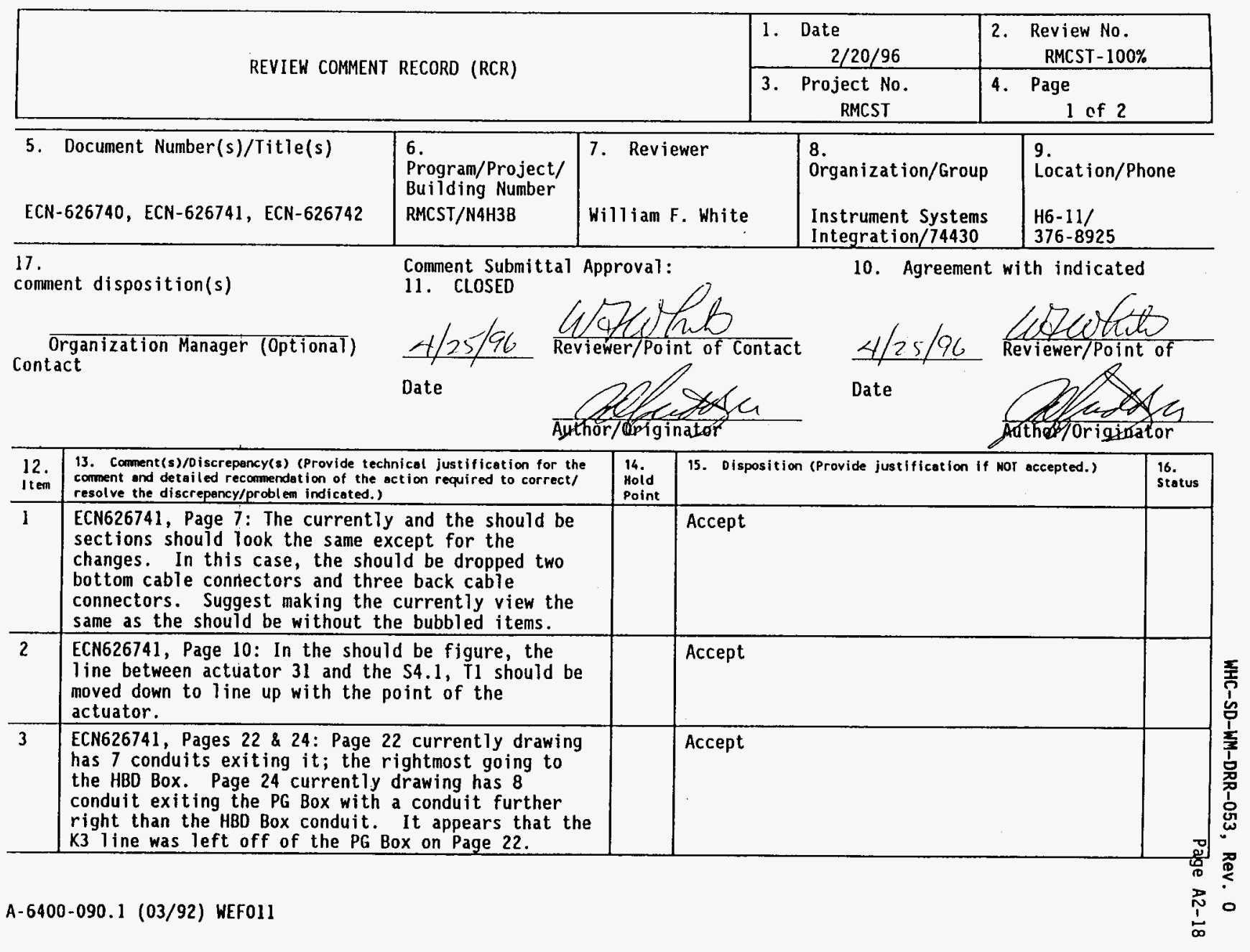




\begin{tabular}{|l|l|}
\hline $\begin{array}{c}\text { 1. } \\
2 / 20 / 96\end{array}$ & $\begin{array}{c}\text { 2. } \\
\text { Review No. } \\
\text { RMCST }-100 \%\end{array}$ \\
\hline $\begin{array}{c}\text { 3. Project No. } \\
\text { RMCST }\end{array}$ & $\begin{array}{c}\text { 4. Page } \\
2 \text { of } 2\end{array}$ \\
\hline
\end{tabular}

4 ECN626741, Page 22: The currently drawing has 7 conduits exiting the bottom of the PG Box, the should be has 8 with the left most one not connected to anything. See the conment above.

5 ECN626741, Pages 15 \& 16: You have changed an 8 input module into a 16 input module. ALL connections to the new module will have to be remarked to the "S2.1-" designation from the "S2-" designation, not just the new additions.

6 ECN626742, Page 3: As you did with the Shield Rcvr Load Cell, above the Grapple Hoist load Cell ADD "SEE H-2-690071 SH 7"

7 ECN626742, Page 5: All References to J6 should be changed to $\mathrm{J6}$ - (for example: "J6v" would become " $J 6-v ")$ - If you change these, you might as well change all "J6" references to "J6-" references.

8 ECN626742, Page 13: For Wire Runs 60 and 62 in the VIA block ADD "SEE NOTE *" under the K16 (60) and $\mathrm{K} 17(62)$.

9 ECN626742, Page 14: Next the K17 Call-out, ADD note flag for note *.

10 ECN626742, Page 17: For run $\mathrm{K} 17$ change the note call-out flag from "e" to "*" or add call-out for flag

11 ECN626742: You show the routing for $\mathrm{K} 17$ but leave out the routing for $K 16$. ADD the $K 16$ routing as well as a note flag call-out for note

\begin{tabular}{|l|l|}
\hline Accept & \\
\hline Accept & \\
\hline Accept & \\
\hline Accept & \\
\hline $\begin{array}{l}\text { Flag notes will be deleted from VIA block } \\
\text { and located as appropriate. }\end{array}$ & \\
\hline Flag notes will be properly located. & \\
\hline Flag notes will be added on that ECN. & \\
\hline Accept & \\
\hline Routing for K16 is shown on ECN 628707. & \\
\hline
\end{tabular}




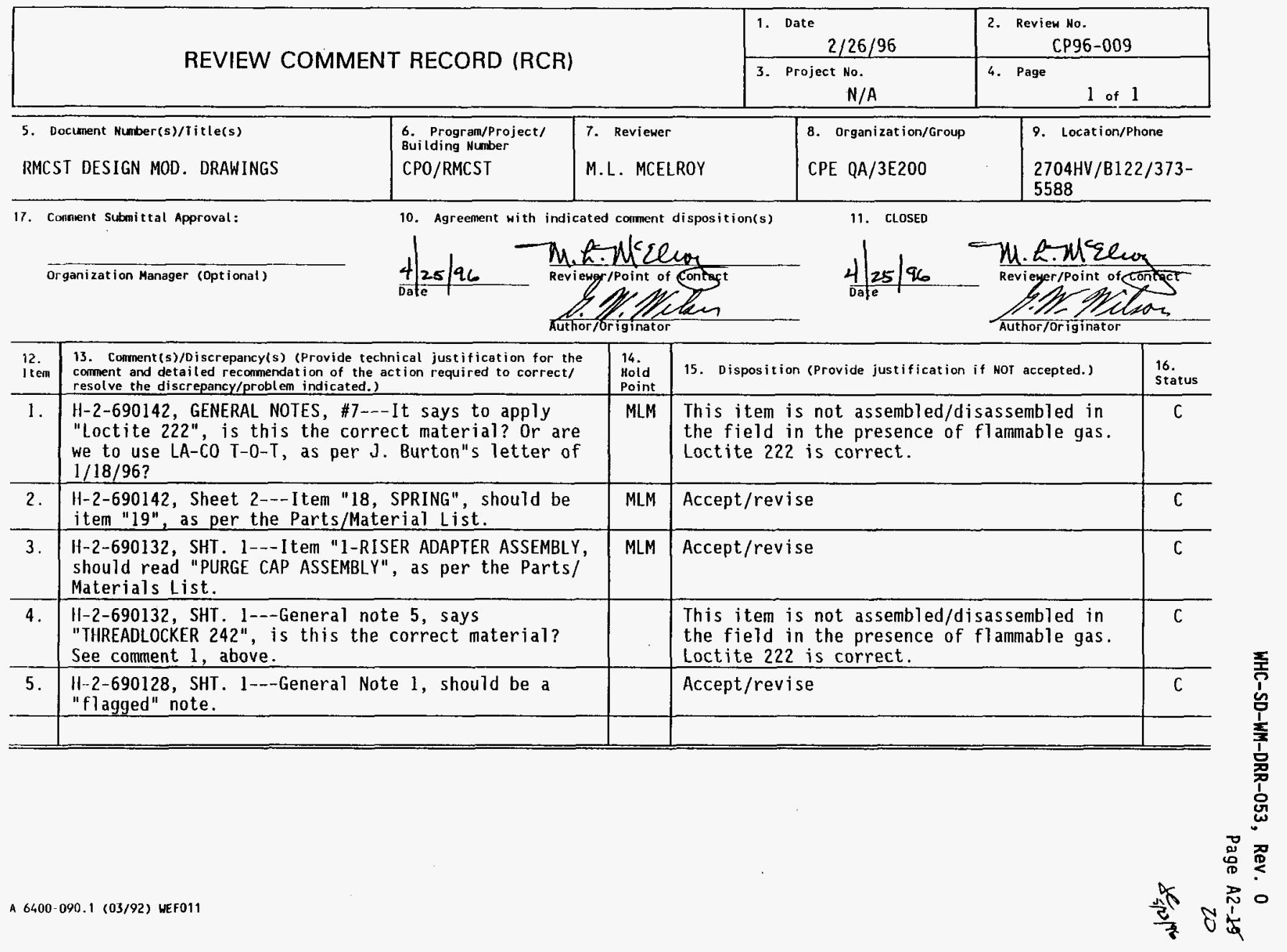




\section{REVIEW COMMENT RECORD (RCR)}

\begin{tabular}{|c|c|}
\hline $\begin{array}{l}\text { 1. Date } \\
\qquad 3 / 12 / 96\end{array}$ & $\begin{array}{r}\text { 2. Review No. } \\
100 \%\end{array}$ \\
\hline $\begin{array}{l}\text { 3. Project No. } \\
\text { ETN-96-003 }\end{array}$ & 4. Page \\
\hline
\end{tabular}

\section{Docunent Number(s)/Title(s)}

See block 13

17. Conrnent Submittal Approval:

Program/Project/ Building Number

Characterization RMCS

10. Agreenent with indicated comment disposition(s)

Organization Manager (Optional)

$$
3 \cdot-12-96
$$$$
\text { Date }
$$

\begin{tabular}{|l|l}
\hline 7. Reviewer & 8. Organization/Group \\
P. J. Martel1 & WCS/ES
\end{tabular}

\section{Ps navtat}

Reviewer/Roint of Eontact

B.P. Mether

Author opiginater
$3 \cdot 12-96$ Date
9. Location/Phone

$M 0255 / 373-5417$

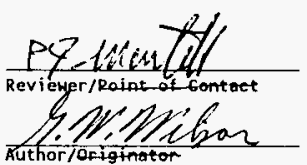

Author/ófiginator

\begin{tabular}{|c|c|c|c|c|}
\hline $\begin{array}{l}12 . \\
1 \text { tein }\end{array}$ & $\begin{array}{l}\text { 13. Comment(s)/Discrepancy(s) (Provide technical justification for the } \\
\text { comment and detailed recomendation of the action required to correct/ } \\
\text { resolve the discrepancy/problem indicated.) }\end{array}$ & $\begin{array}{l}14 . \\
\text { Hoid } \\
\text { Point }\end{array}$ & 15. Disposition (Provide justification if NOT accepted.) & $\begin{array}{l}16 . \\
\text { Status }\end{array}$ \\
\hline & $\begin{array}{l}\text { The following documents were reviewed in support of } \\
\text { the core sample truck, flammable gas modifications } \\
\text { final design review: ECN\# } 628706 \text { through } 628715 \text {, } \\
628717,628720, \& 626740 \text { through } 626742 \text {; and } \mathrm{H}-2 \\
\text { drawing\# } 690128,690131,690132, \& 690142 \text {. } \\
\text { No comments/discrepancies exist that would impact } \\
\text { environmental approval of the described design. }\end{array}$ & none & none & closed \\
\hline & & & & \\
\hline & & & & \\
\hline & & & & \\
\hline & & & & \\
\hline & & & & \\
\hline & & & & \\
\hline & & & & \\
\hline & & & & \\
\hline
\end{tabular}




\section{REVIEW COMMENT RECORD (RCR)}

\begin{tabular}{|l|l|}
\hline $\begin{array}{c}\text { 1. Date } \\
3 / 12 / 96\end{array}$ & $\begin{array}{l}\text { 2. Review No. } \\
100 \%\end{array}$ \\
\hline $\begin{array}{c}\text { 3. Project No. } \\
\text { ETN-96-003 }\end{array}$ & 4. Page \\
\hline
\end{tabular}

5. Document Number(s)/Title(s)

See block 13

17. Comment Submittal Approval:

Organization Manager (Optional)

6. Program/Project/ Building Number

Characterization RMCS

10. Agreement with indicated comment disposition(s)

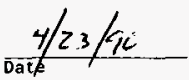

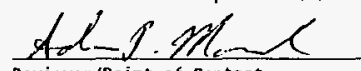
Revigyer/Point of Lataet

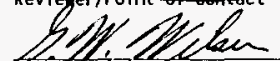
Authoforianator

\begin{tabular}{l|l} 
7. Reviewer & 8. Organization/Group \\
A. P. Mousel & TCP/Field Eng.
\end{tabular}

9. Locat i on/Phone

2704HV/373-9207

11. CLOSED
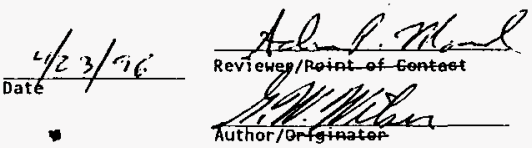

14.

12. 13. Comment(s)/Discrepancy(s) (Provide technical justification for the comment and detailed recomalendation of the action
resolve the discrepancy/problem indicated.)

The following documents were reviewed in support of

the core sample truck, flammable gas modifications

final design review: Design Review Checklist;

ECN\# 628706 through $628715,628717,628720$, \&

626740 through 626742; and

H-2 drawing\# $690128,690131,690132, \& 690142$.

No comments/discrepancies exist that would impact

field engineering approval of the described design.

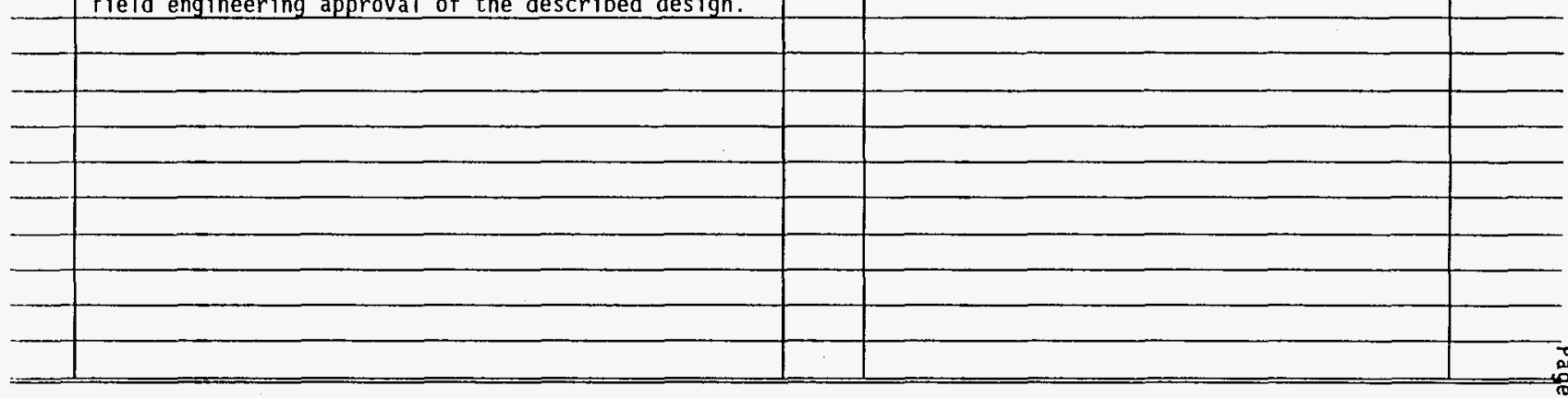

A $6400-090.1(03 / 92)$ WEF011

Hold

15. Disposition (Provide justification if NOT accepted.) none none Status closed 


\begin{tabular}{|c|c|c|}
\hline \multirow{2}{*}{ REVIEW COMMENT RECORD (RCR) } & $\begin{array}{l}\text { 1. Date } \\
3 / 12 / 96 \\
\end{array}$ & $\begin{array}{r}\text { 2. Review Ho. } \\
100 \% \\
\end{array}$ \\
\hline & $\begin{array}{l}\text { 3. Project No. } \\
\text { ETN-96-003 }\end{array}$ & 4. Page \\
\hline
\end{tabular}

5. Document Number(s)/Title(s)

See block 13

17. Comment Submittal Approval:

Organization Manager (Optional)

\begin{tabular}{|c|c|}
\hline $\begin{array}{l}\text { 6. Program/Project, } \\
\text { Building Number }\end{array}$ & 7. Reviewer \\
\hline $\begin{array}{l}\text { Characterization } \\
\text { RMCS }\end{array}$ & L. S. Krogsrud \\
\hline
\end{tabular}

10. Agreement with indicated comment disposition(s)
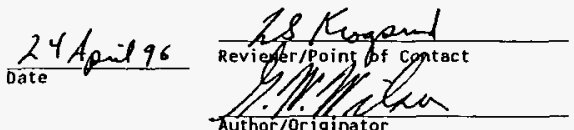

8. Organization/Group

TWRS Safety

9. Location/Phone

$2751 \mathrm{E} / 372-2302$
11. CLIOSED

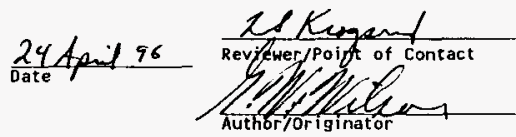

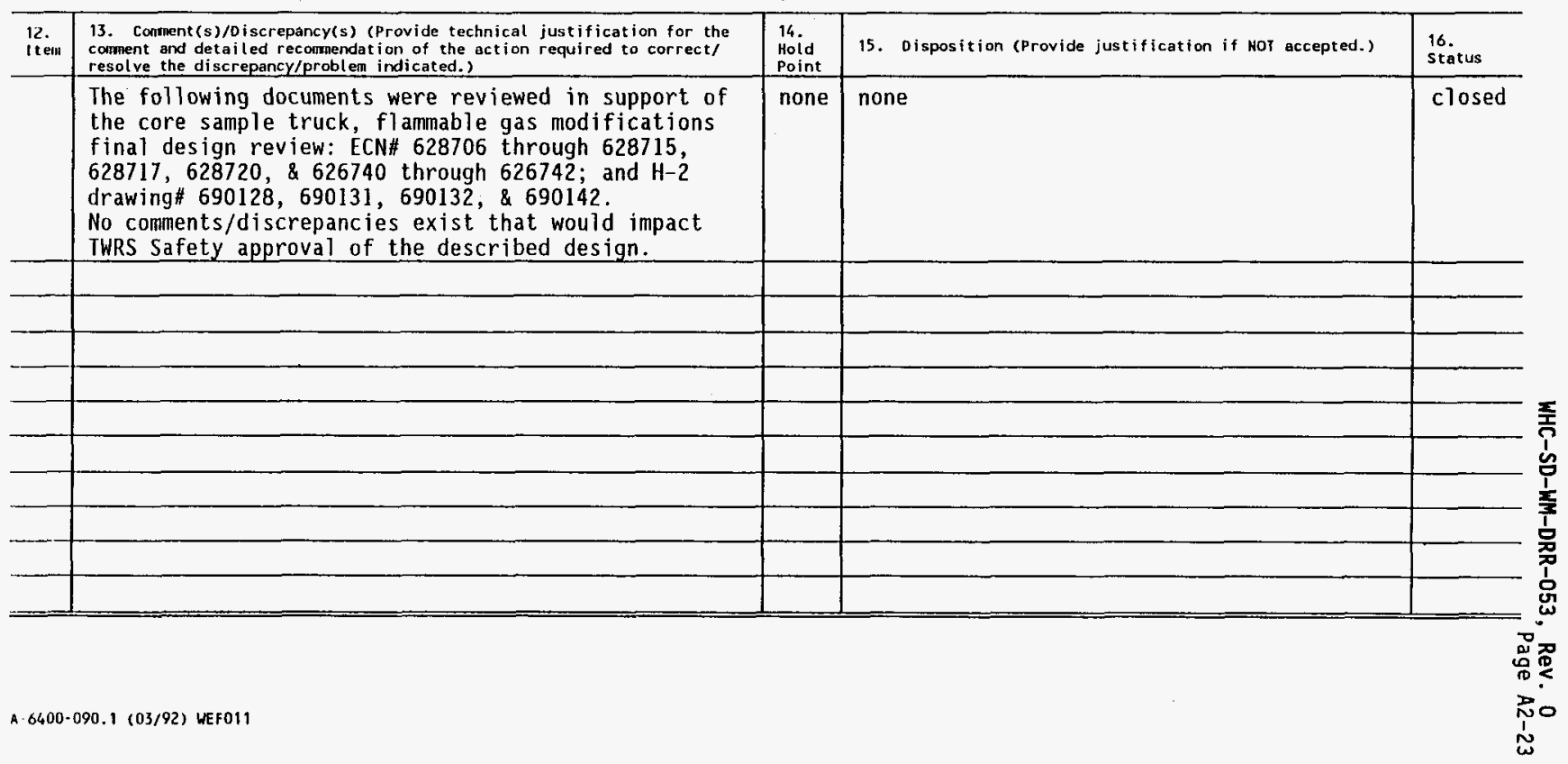




\begin{tabular}{|c|l|l|}
\hline \multirow{2}{*}{ REVIEW COMMENT RECORD (RCR) } & $\begin{array}{l}\text { 1. Date } \\
4 / 26 / 96\end{array}$ & $\begin{array}{l}\text { Review Ho. } \\
\text { WFW-964Q-1 }\end{array}$ \\
\cline { 2 - 4 } & $\begin{array}{l}3 . \text { Project No. } \\
\text { Core Sample Trucks }\end{array}$ & 4. Page \\
\hline
\end{tabular}

5. Document Number(s)/Title(s)

Engineering Change Notice 623775

17. Comment Submittal Approval: 6. Program/Project/ Building Number 200 General

William f. White

10. Agreement with indicated comment dispositiog(s)

Organization Manager (Optional)

$$
5 / 7 / 96
$$

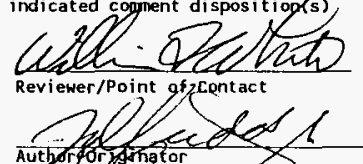

B. Organization/Graup

Instrument Systems Integration $/ 74430$

9. Location/Phone

H6-11/

376-8925

11. CLOSED $\frac{5 / 7 / 96}{\text { Date }}$ $-$ Reviewer/Point of 'Cont act

Autjorforysorator

\begin{tabular}{|c|c|c|c|c|}
\hline $\begin{array}{l}12 . \\
1 \text { tein }\end{array}$ & $\begin{array}{l}\text { 13. Comment(s)/Discrepancy(s) (Provide technical justification for the } \\
\text { conment and detailed recommendation of the action required to correct/ } \\
\text { resolve the discrepancy/problem indicated.) }\end{array}$ & $\begin{array}{l}\text { P4. } \\
\text { Mold } \\
\text { Point }\end{array}$ & 15. Disposition (Provide justification if NOT accepted.) & $\begin{array}{l}16 . \\
\text { Status }\end{array}$ \\
\hline 1 & $\begin{array}{l}\text { GENERAL: } \\
\text { Several of the logic diagrams are extremely hard to } \\
\text { read. Put them on } 11 \times 17 \text { inch pages and they would be } \\
\text { readable. }\end{array}$ & & $\begin{array}{l}\text { Noted: that is more a function of Word } \\
\text { Perfect than anything else; have been trying } \\
\text { to find a way to fix it. }\end{array}$ & \\
\hline 2 & $\begin{array}{l}\text { Page } 1: \\
\text { Block } 11 b \text { needs the Work Package Number. }\end{array}$ & & Provided by JD Criddle Jr organization. & \\
\hline 3 & $\begin{array}{l}\text { Page 1: } \\
\text { Block } 12 \text { needs the USQE number on the first line } \\
\text { along with the ETN. }\end{array}$ & & Provided by JD Criddle Jr organization. & \\
\hline 4 & $\begin{array}{l}\text { Page } 1: \\
\text { Block } 13 b-1 \text { suggest you add a reference to the } S A \\
\text { as additional justification for the change. }\end{array}$ & & Provided by JD Criddle Jr organization. & \\
\hline 5 & $\begin{array}{l}\text { Page } 3: \\
\text { Contact } 32 \text { FUNCTION currently reads "FUTURE USE" - } \\
\text { need to CHANGE this to read "PENETRATION RATE LOW } \\
\text { ALARM LIGHT" }\end{array}$ & & Accept & \\
\hline 6 & $\begin{array}{l}\text { Page } 3 \text { : } \\
\text { Contact } 5 \text { should ADO } 1 \text { ines } 444,486 \text {, and } 473 \text { (not } \\
545 \text { ) and it should also REMOVE } 7 \text { ines } 259 \text { and } 264 \text {. }\end{array}$ & & Accept & \\
\hline
\end{tabular}




\section{REVIEW COMMENT RECORD (RCR)}

\begin{tabular}{|l|l|}
\hline $\begin{array}{l}\text { 1. Date } \\
4 / 26 / 96\end{array}$ & $\begin{array}{l}\text { 2. Review No. } \\
\text { WFW-964Q-1 }\end{array}$ \\
\hline $\begin{array}{l}\text { 3. Project No. } \\
\text { Core Sample Trucks }\end{array}$ & 4. Page \\
2 of 5 \\
\hline
\end{tabular}

\begin{tabular}{|c|c|c|c|c|}
\hline $\begin{array}{l}12 . \\
\text { item }\end{array}$ & $\begin{array}{l}\text { 13. Corment(s)/Discrepancy(s) (Provide technical justification for the } \\
\text { comment and detailed recommendation of the action required to correct/ } \\
\text { resolve the discrepancy/problem indicated.) }\end{array}$ & $\begin{array}{l}\text { 14. } \\
\text { Hoid } \\
\text { Point }\end{array}$ & 15. Disposition (Provide justification if NOr accepted.) & $\begin{array}{l}16 . \\
\text { status }\end{array}$ \\
\hline 7 & $\begin{array}{l}\text { Page } 3 \text { : } \\
\text { Contact } 30 \text { should read " }<49,395>\text { " rather than " }<49> \\
395 " \text { the way this change would be incorporated into } \\
\text { the drawing. }\end{array}$ & & Accept & \\
\hline 8 & $\begin{array}{l}\text { Page } 3 \text { : } \\
\text { Contact } 31 \text { should read " }<50,364>"\end{array}$ & & Accept & \\
\hline 9 & $\begin{array}{l}\text { Page 3: } \\
\text { Contact } 32 \text { should read " }<51,495>\text { " }\end{array}$ & & Accept & \\
\hline 10 & $\begin{array}{l}\text { Page } 3: \\
\text { Contact } 40-\text { CHANGE "176" to " } 177 "\end{array}$ & . & Accept & \\
\hline 11 & $\begin{array}{l}\text { Page } 3: \\
\text { Contact } 52 \text { - CHANGE "176" to "I77" }\end{array}$ & & Accept & \\
\hline 12 & $\begin{array}{l}\text { Page 3: } \\
\text { Contact } 66 \text { should read " }<89,398>" \text {. }\end{array}$ & & Accept & \\
\hline 13 & $\begin{array}{l}\text { Page 3: } \\
\text { Contact } 73 \text { - CHANGE " } 216 " \text { to read " } 221 \text { " and ADD } 373 \\
\text { and } 375 \text { to the listing. }\end{array}$ & & Accept & \\
\hline 14 & $\begin{array}{l}\text { Page 4: } \\
\text { For contact } 4 \text { you list "S43a", yet looking at the } \\
\text { drawing on sheet } 3 \text {, there does not seem to be a } \\
\text { difference between } S 40, S 41, S 42 \text { and } S 43 \text {. Do these } \\
\text { all need to be "a"? }\end{array}$ & & Accept, will remove "a". & \\
\hline 15 & $\begin{array}{l}\text { Page } 6: \\
\text { For contacts } 114 \text { and } 115 \text {, these need to be changed } \\
\text { to be "40A" and "40B" to be consistant with page } 14 \text {. }\end{array}$ & & Accept & \\
\hline 16 & $\begin{array}{l}\text { Page } 7 \text { : } \\
4 \text { th } 1 \text { ine from the bottom - CHANGE "contact number } \\
372, \ldots \text { to read "contact number } 370, \ldots \text { ".." }\end{array}$ & & Accept & \\
\hline 17 & $\begin{array}{l}\text { Page } 7 \text { : } \\
\text { For contact } 215 \text {, in addition to those given, ADD } \\
1 \text { ines } 259 \text { and } 264 \text { to the listing. }\end{array}$ & & Accept & \\
\hline
\end{tabular}

A $6400-090.1$ (03/92) WEF011 


\begin{tabular}{|c|l|l|}
\hline \multirow{2}{*}{ REVIEW COMMENT RECORD (RCR) } & $\begin{array}{l}\text { 1. Date Review No. } \\
\text { WFW-964Q-1 }\end{array}$ \\
\cline { 2 - 3 } & $\begin{array}{l}\text { 3. Project No. } \\
\text { Core Sample Trucks }\end{array}$ & $\begin{array}{l}\text { 4. Page } \\
3 \text { of } 5\end{array}$ \\
\hline
\end{tabular}

\begin{tabular}{|c|c|c|c|c|}
\hline $\begin{array}{l}12 . \\
\text { 1tem }\end{array}$ & $\begin{array}{l}\text { 13. Comment(s)/oiscrepancy(s) (Provide technical justification for the } \\
\text { comment and detailed recommendation of the action required to correct/ } \\
\text { resolve the discrepancy/problem indicated.) }\end{array}$ & $\begin{array}{l}\text { 14. } \\
\text { Hoid } \\
\text { Point }\end{array}$ & 15. Disposition (Provide justification if NOI accepted.) & $\begin{array}{l}16 . \\
\text { status }\end{array}$ \\
\hline 18 & $\begin{array}{l}\text { Page } 7: \\
\text { For contact } 216, \text { in addition to those given, ADD } \\
\text { line } 481 \text { to the } 7 \text { isting. }\end{array}$ & & Accept & \\
\hline 19 & $\begin{array}{l}\text { Page } 7: \\
\text { For Contact } 320 \text {, REMOVE } 1 \text { ine } 305 .\end{array}$ & & Accept & \\
\hline 20 & $\begin{array}{l}\text { Page } 8: \\
\text { For contact } 301 \text {, ADD line } 389 .\end{array}$ & & Accept & \\
\hline 21 & $\begin{array}{l}\text { Page 8: } \\
\text { Contact } 341 \text { - CHANGE " } 541 \text { " to " } 542 " \text { (see ECN page } \\
31 \text { ). }\end{array}$ & & Accept & \\
\hline 22 & $\begin{array}{l}\text { Page } 8 \text { : } \\
\text { For contacts } 354 \text { and } 355 \text { - CHANGE " } 464 \text { " to " } 472 \text { " }\end{array}$ & & Accept & \\
\hline 23 & $\begin{array}{l}\text { Page } 8: \\
\text { For contact } 355 \text { ADD line } 479 .\end{array}$ & & Accept & \\
\hline 24 & $\begin{array}{l}\text { Page } 8: \\
\text { For contact } 220 \text {, CHANGE " } 428 \text { " to " } 482 \text { " }\end{array}$ & & Accept & \\
\hline 25 & $\begin{array}{l}\text { Page } 8: \\
\text { For contact } 223 \text {, CHANGE " } 626 " \text { to " } 625 "\end{array}$ & & Accept & \\
\hline 26 & $\begin{array}{l}\text { Page } 10: \\
\text { For contact } 772 \text {, CHANGE "626" to "625, 628" }\end{array}$ & & Accept & \\
\hline 27 & $\begin{array}{l}\text { Page } 10: \\
\text { For contact } 773 \text {, CHANGE " } 627 " \text { to } " 626,629,631 "\end{array}$ & & Accept & \\
\hline 28 & $\begin{array}{l}\text { Page } 13: \\
\text { The module in slot } 2 \text { is the same as the module in } \\
\text { slot } 3 \text {. Why are they wired differently? }\end{array}$ & & $\begin{array}{l}\text { Response: the } 8 \text { new inputs added to slot } 2 \\
\text { are tied (via the various switches) to } 48- \\
\mathrm{H} 1 \text {. The corresponding neutral is } 49-\mathrm{N} \text {. (See } \\
\text { pg } 14 \text { of the ECN). This is not the case for } \\
\text { the module in slot } 3 \text {. }\end{array}$ & \\
\hline
\end{tabular}




\begin{tabular}{|c|c|c|}
\hline \multirow{2}{*}{ REVIEW COMMENT RECORD (RCR) } & \begin{tabular}{rl|}
1. Date \\
& $4 / 26 / 96$ \\
\end{tabular} & $\begin{array}{l}\text { 2. Review No. } \\
\text { WFW-964Q-1 }\end{array}$ \\
\hline & $\begin{array}{l}\text { 3. Project No. } \\
\text { Core Sample Trucks }\end{array}$ & 4. Page 4 of 5 \\
\hline
\end{tabular}

\begin{tabular}{|c|c|c|c|c|}
\hline $\begin{array}{l}12 . \\
\text { Itein }\end{array}$ & $\begin{array}{l}\text { 13. Comnent(s)/Discrepancy(s) (Provide technical justification for the } \\
\text { comment and detailed recommendat ion of the action required to correct/ } \\
\text { resolve the discrepancy/problem indicated.) }\end{array}$ & $\begin{array}{l}14 . \\
\text { Hoid } \\
\text { Point }\end{array}$ & 15. Disposition (Provide justification if NOT accepted.) & $\begin{array}{l}16 . \\
\text { Status }\end{array}$ \\
\hline 29 & $\begin{array}{l}\text { Page 14: } \\
\text { [This is an example of hard to read!] The "SHOULD } \\
\text { BE" drawing includes the title for } 7 \text { ine } 34 \text { that was } \\
\text { changed on page } 12 \text { of this ECN but is unchanged } \\
\text { here. Need them to be consistant. }\end{array}$ & & Accept; will revise this page of the ECN. & \\
\hline 30 & $\begin{array}{l}\text { Page 12: } \\
\text { The first group of changes would el iminate the } \\
\text { "(NOTE 8) " that follows the existing text. I am } \\
\text { pretty sure you want to keep the reference to note } \\
8 \text {. If you do, within each of the quotes, ADD "(NOTE } \\
8 \text { ". }\end{array}$ & & Accept; will keep reference to note 8 . & \\
\hline 31 & $\begin{array}{l}\text { Page 22: } \\
\text { For the third group of changes on this page, I } \\
\text { suggest adding the line numbers for these changes to } \\
\text { make them easy to find (They are } 346,322 \text {, and } 326 \\
\text { respectively). }\end{array}$ & & Accept & \\
\hline 32 & $\begin{array}{l}\text { Page } 30 \text { : } \\
\text { In the sketch, the line joining the three legs is } \\
\text { extended downward. Where are the other contacts for } \\
\text { this alarm? }\end{array}$ & & Accept; will remove errant line. & \\
\hline 33 & $\begin{array}{l}\text { Page } 31 \text { : } \\
\text { CHANGE "SINGE" to "SINGLE" }\end{array}$ & & Accept & \\
\hline 34 & $\begin{array}{l}\text { Page 42: } \\
\text { The line numbers and the contacts do not line up } \\
\text { properly. Line } 625 \text { should have } 772 \text { and }\langle 223\rangle ; \text { Line } \\
626 \text { should have } 773 ; \text { Line } 628 \text { should have } 772 \text { and } \\
<224\rangle ; \text { Line } 629 \text { should have } 773 ; \text { and Line } 631 \text { should } \\
\text { have } 773 \text { and }\langle 2341\rangle \text {. }\end{array}$ & & Accept & \\
\hline 35 & $\begin{array}{l}\text { H-2-690069, Sheet } 5 \text {, Zone C4 \& D4: } \\
\text { Lines } 110 \text { through } 115 \text { have wire run numbers that } \\
\text { start with a "C" }- \text { Should these start with a "0"? }\end{array}$ & & Response: "C" prefix is correct as shown. & \\
\hline
\end{tabular}




\begin{tabular}{|c|c|c|}
\hline \multirow{2}{*}{ REVIEW COMMENT RECORD (RCR) } & $\begin{array}{l}\text { 1. Date } \\
4 / 26 / 96\end{array}$ & $\begin{array}{l}\text { 2. Review No. } \\
\text { WFW-964Q-1 }\end{array}$ \\
\hline & $\begin{array}{l}\text { 3. Project No. } \\
\text { Core Sample Trucks }\end{array}$ & $\begin{array}{l}\text { 4. Page } 5 \text { of } 5 \\
\text { nat }\end{array}$ \\
\hline
\end{tabular}

\begin{tabular}{|c|c|c|c|c|}
\hline $\begin{array}{l}12 . \\
1 \text { tem }\end{array}$ & $\begin{array}{l}\text { 13. Conment(s)/0iscrepancy(s) (Provide technical justification for the } \\
\text { comment and detailed recommendation of the action required to correct/ } \\
\text { resolve the discrepancy/problem indicated.) }\end{array}$ & $\begin{array}{l}\text { 14. } \\
\text { Hold } \\
\text { Point }\end{array}$ & 15. Disposition (Provide justification if NOT accepted.) & $\begin{array}{l}16 . \\
\text { status }\end{array}$ \\
\hline 36 & $\begin{array}{l}\text { H-2-690069, Sheet } 6 \text {, lines } 138 \text { \& } 139 \text { : } \\
\text { Just a side note these two lines are logically the } \\
\text { same as having } 205 \text { and } 206 \text { in parallel followed by } \\
\text { the series contact of } 216 \text {. THIS IS NOT A COMMENT FOR } \\
\text { A CHANGE, IT IS JUST A NOTE. }\end{array}$ & & Noted & \\
\hline 37 & $\begin{array}{l}\text { H-2-690069, Sheet } 6 \text {, Zone D4, Line } 152: \\
\text { Since changing other locations (Lines } 156 \text { \& } 159 \text { ) } \\
\text { from "206" to "214", should the contact in this } \\
\text { location also be "214"? }\end{array}$ & & $\begin{array}{l}\text { Response: either way is functionally the } \\
\text { same. }\end{array}$ & \\
\hline
\end{tabular}




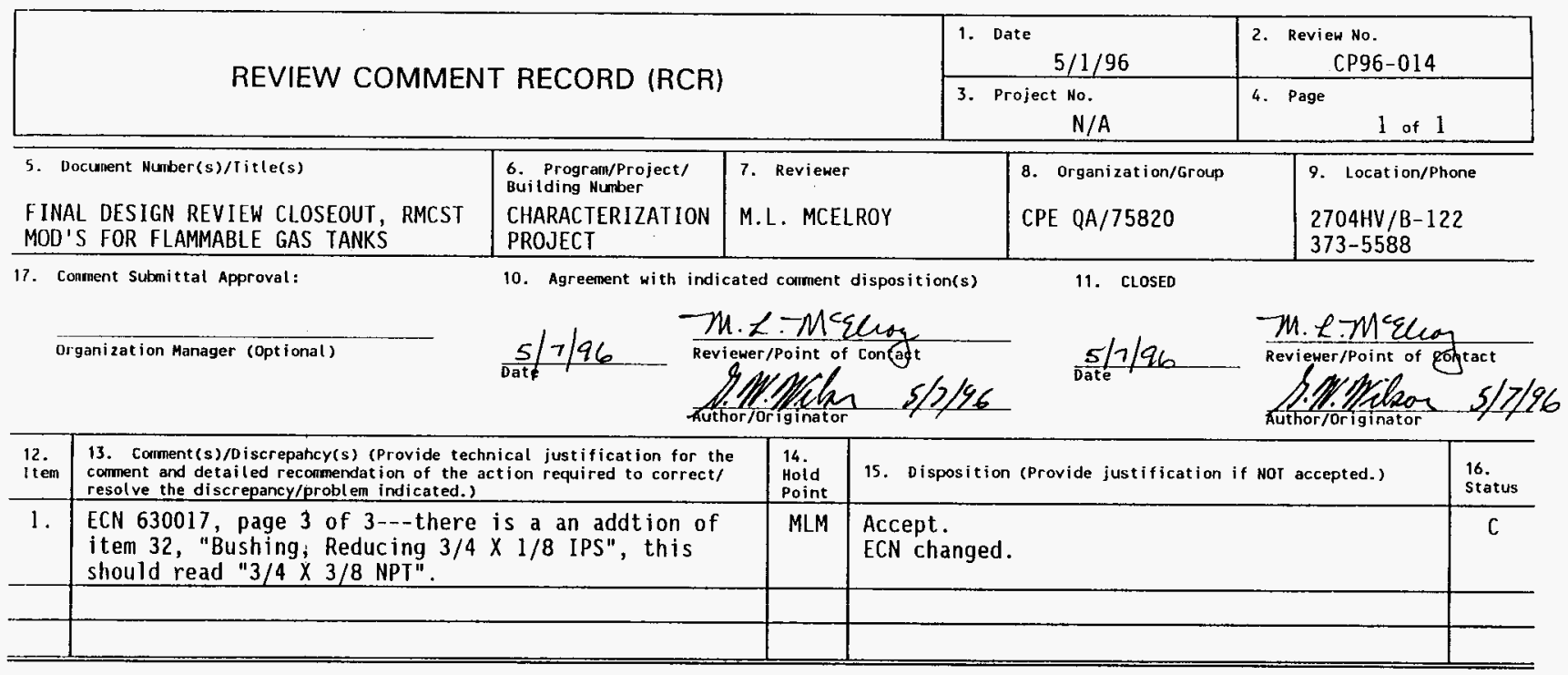




\begin{tabular}{|c|l|l|l|}
\hline \multirow{2}{*}{ REVIEW COMMENT RECORD (RCR) } & $\begin{array}{l}\text { 1. Date } \\
\text { May } 1,1996\end{array}$ & $\begin{array}{l}\text { Review No. } \\
484-96\end{array}$ \\
\cline { 2 - 3 } & $\begin{array}{l}\text { 3. Project No. } \\
\text { Characterization }\end{array}$ & $\begin{array}{l}4 . \text { Page } \\
1 \text { of } 1\end{array}$ \\
\hline
\end{tabular}

\section{Document Number(s)/Title(s)}

Drawings $\mathrm{H}-2-690134, \mathrm{H}-2-690136 /$ RMCST Dril1 String Arrangements.

17. Comnent Submittal Approval:

Organization Manager (Optional

6. Programproject/ Building Number

Characterization
8. Organization/Group TWRS-Nuclear Safety
9. Location/Phone

2751E/F111/22322
10. Agreement with indicated comment disposition(s)

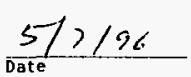

m. $\theta$ man Inl. Revigmer/Point of Contact Revigger/Point of hatharioriginator
11. CLOSED

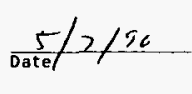

$m \cdot t$ m Trat Revigwer/Point of Contact

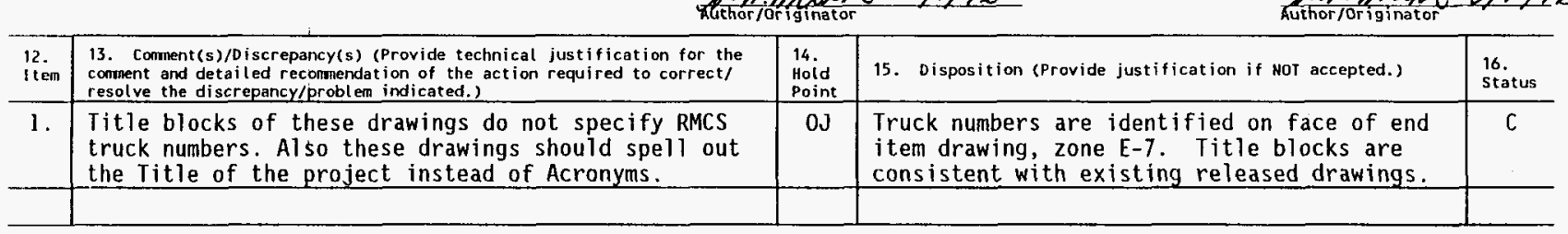




\section{REVIEW COMMENT RECORD (RCR)}

\begin{tabular}{|l|l|}
\hline $\begin{array}{l}\text { 1. Date } \\
05 / 04 / 96\end{array}$ & 2. Review No. \\
\hline $\begin{array}{c}\text { 3. Project No. } \\
\text { Characterization } \\
\text { Project - RMCS Trucks } \\
\text { 3 and 4 }\end{array}$ & 4. Page \\
\hline
\end{tabular}

5. Document Number(s)/Title(s)

ECN 623775

IECN 630017 Ding $\begin{aligned} & \mathrm{H}-2-690134 \\ & \mathrm{H}-2-690136\end{aligned}$

17. Connent Submittal Approval:

\begin{tabular}{l|l}
$\begin{array}{l}\text { 6. Program/Project/ } \\
\text { Building Number }\end{array}$ & 7. Reviener \\
Characterization & K. S. Tollefson \\
Project RMCS & \\
Trucks $3 \& 4$ &
\end{tabular}

10. Agreement with indicated comment disposition(s)

8. Organization/Group
TWRS
Characterization
Project -
Environmental
Compliance

9. Location/Phone

2704 HV/B-119

$372-2234$
Organization Manager (Optional)

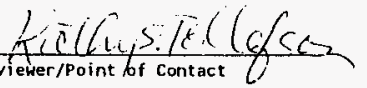

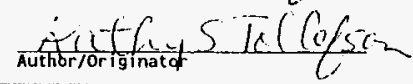

11. CLOSED

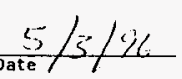

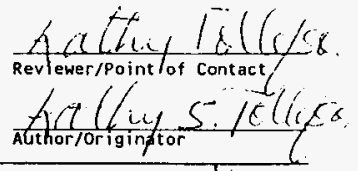
Revewer/Point of contact Althor/origingtor $5 \cdot 16)$

\begin{tabular}{l|l|l|}
\hline 12. & $\begin{array}{l}\text { 13. Comment (s)/oiscrepancy(s) (Provide technical justification for the } \\
\text { 1 temment and detai ied recommendat ion of the act ion required to correct/ } \\
\text { resolve the discrepancy/problem indicated.) }\end{array}$ \\
\hline 1 & No comment response \\
\hline & & \\
\hline & & \\
\hline & \\
\hline & \\
\hline
\end{tabular}

\begin{tabular}{|l|l|l|}
$\begin{array}{l}\text { M6. } \\
\text { Hoid } \\
\text { Point }\end{array}$ & 15. Oisposition (Provide justification if NOT accepted.) & $\begin{array}{l}16 . \\
\text { status }\end{array}$ \\
\hline & & \\
\hline & & \\
\hline & & \\
\hline & & \\
\hline & & \\
\hline & & \\
\hline
\end{tabular}




\begin{tabular}{|l|l|l|}
\hline \multirow{2}{*}{ REVIEW COMMENT RECORD (RCR) } & 1. 0ate & $5-6-96$ \\
\cline { 2 - 3 } & 3. Project No. & 4. Page \\
\hline
\end{tabular}

5. Document Mumber(s)/Iitle(s)
ECN 630017

17. Conment Submittal Approval:

Organization Manager (Optional)

\begin{tabular}{|l|l|}
$\begin{array}{l}\text { 6. Program/Project/ } \\
\text { Building Number } \\
200 \mathrm{G}\end{array}$ & $\begin{array}{l}\text { 7. Reviewer } \\
\text { R.Y. Seda }\end{array}$ \\
\hline
\end{tabular}

10. Agreement with indicated comment djposition(s)
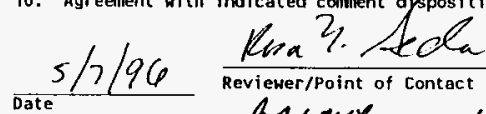

Reviewer/Point of Contact

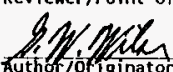

\begin{tabular}{|l|l|}
\hline $\begin{array}{l}\text { 8. Organization/Group } \\
75250\end{array}$ & $\begin{array}{l}\text { 9. Location/Phone } \\
\text { ETCl/3-0061 }\end{array}$ \\
\hline
\end{tabular}

11. CLOSED
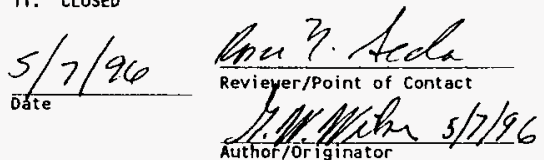

\begin{tabular}{|c|c|c|c|c|}
\hline item & $\begin{array}{l}\text { 13. Comment(s)/Discrepancy(s) (Provide technical justification for the } \\
\text { comnent and detailed recommendation of the action required to correct/ } \\
\text { resolve the discrepancy/problem indicated.) }\end{array}$ & $\begin{array}{l}14 . \\
\text { Hoid } \\
\text { Point }\end{array}$ & 15. Disposition (Provide justification if Nor accepted.) & $\begin{array}{l}16 . \\
\text { status }\end{array}$ \\
\hline 1 & $\begin{array}{l}\text { On block 18, the operating procedures were } \\
\text { ident ified. The instruction on block } 18 \text { indicate } \\
\text { that the affected document has to be entered in } \\
\text { block } 19 \text {. Block } 19 \text { indicates N/A. Please correct } \\
\text { this and indicate the affected document. }\end{array}$ & & Accept, ECN corrected. & $\mathrm{c}$ \\
\hline 2 & $\begin{array}{l}\text { On page 3, Part No. } 16 \text { was deleted. On } \mathrm{H}-2-91671 \\
\text { general note } 10 \text { states "The pressure gauge adapter } \\
\text { can be used to measure tank pressure by inserting } \\
\text { into PN } 16 \text { and reading pressure gauge when gauge is } \\
\text { in vertical position". Please change note } 10 \text { to } \\
\text { reflect deletion of PN } 16 \text {. }\end{array}$ & & Accept, note and reference deleted by ECN. & C \\
\hline & & & & \\
\hline & & & & \\
\hline & & & & \\
\hline & & & & \\
\hline & & & & \\
\hline & & & & \\
\hline & & & & \\
\hline & & & & \\
\hline
\end{tabular}




\begin{tabular}{|c|c|c|}
\hline \multirow{2}{*}{ REVIEW COMMENT RECORD (RCR) } & $\begin{array}{l}\text { 1. Date } \\
5-6-96\end{array}$ & 2. Review No. \\
\hline & 3. Project No. & 4. Page \\
\hline
\end{tabular}

5. Docunient Number(s)/Title(s)

11-2-690134 Dri11 String Arrangements (RMCST)

17. Conment Submittal Approval:

6. Progran/Project/ Building Humber

$200 G$

10. Agreement with indicated comment disposition(s)

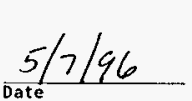
Rosk 7. Lede Revifwer/Point of Contact

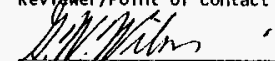

Author/friginator
8. Organization/Group

75250

9. Location/Phone

ETC1/3-0061

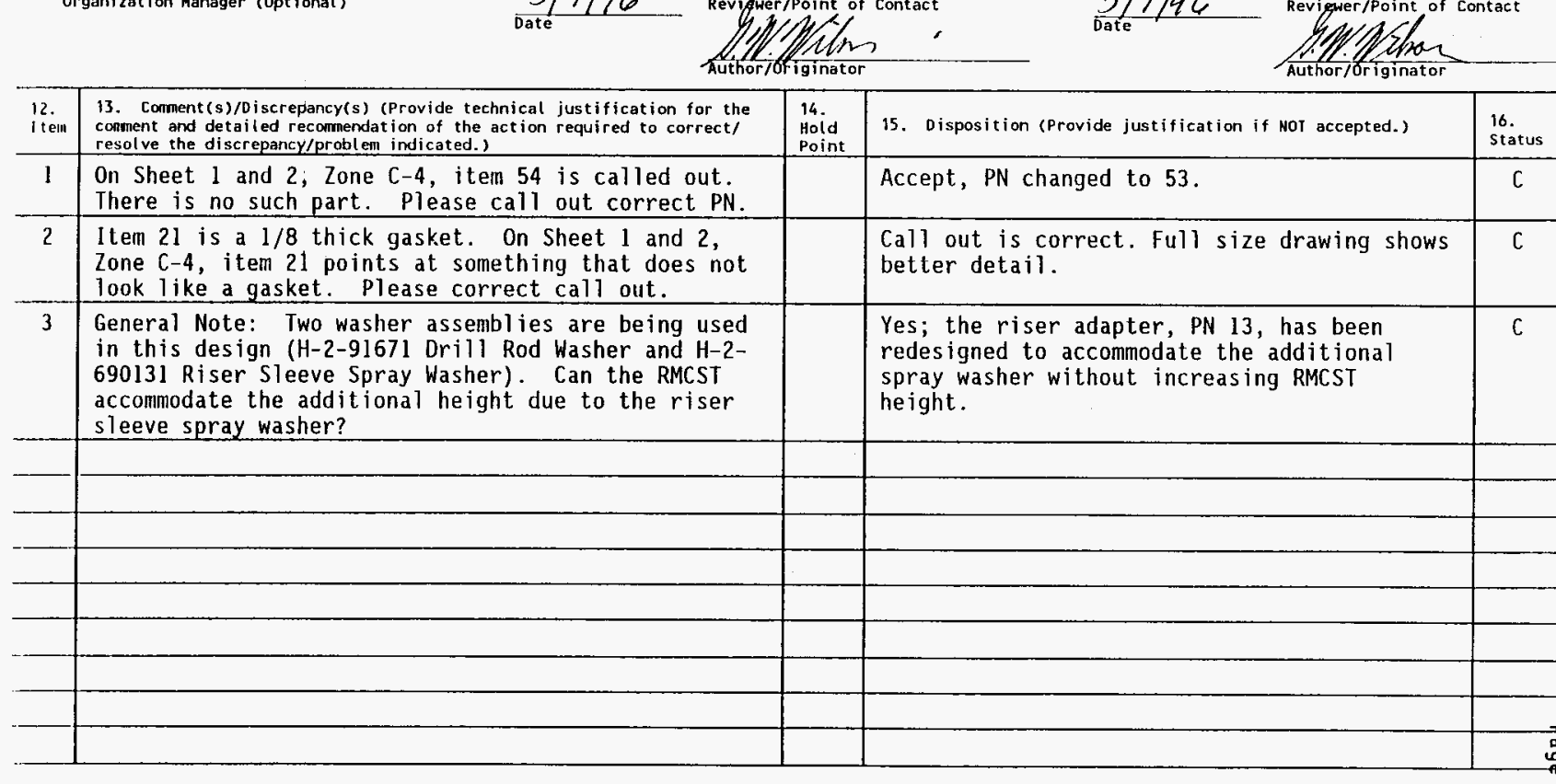

11. CLOSED
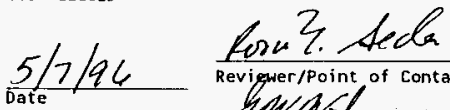

Revigwer/Point of Contact
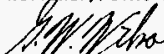

(6400-090.1 (03/92) HEF011 


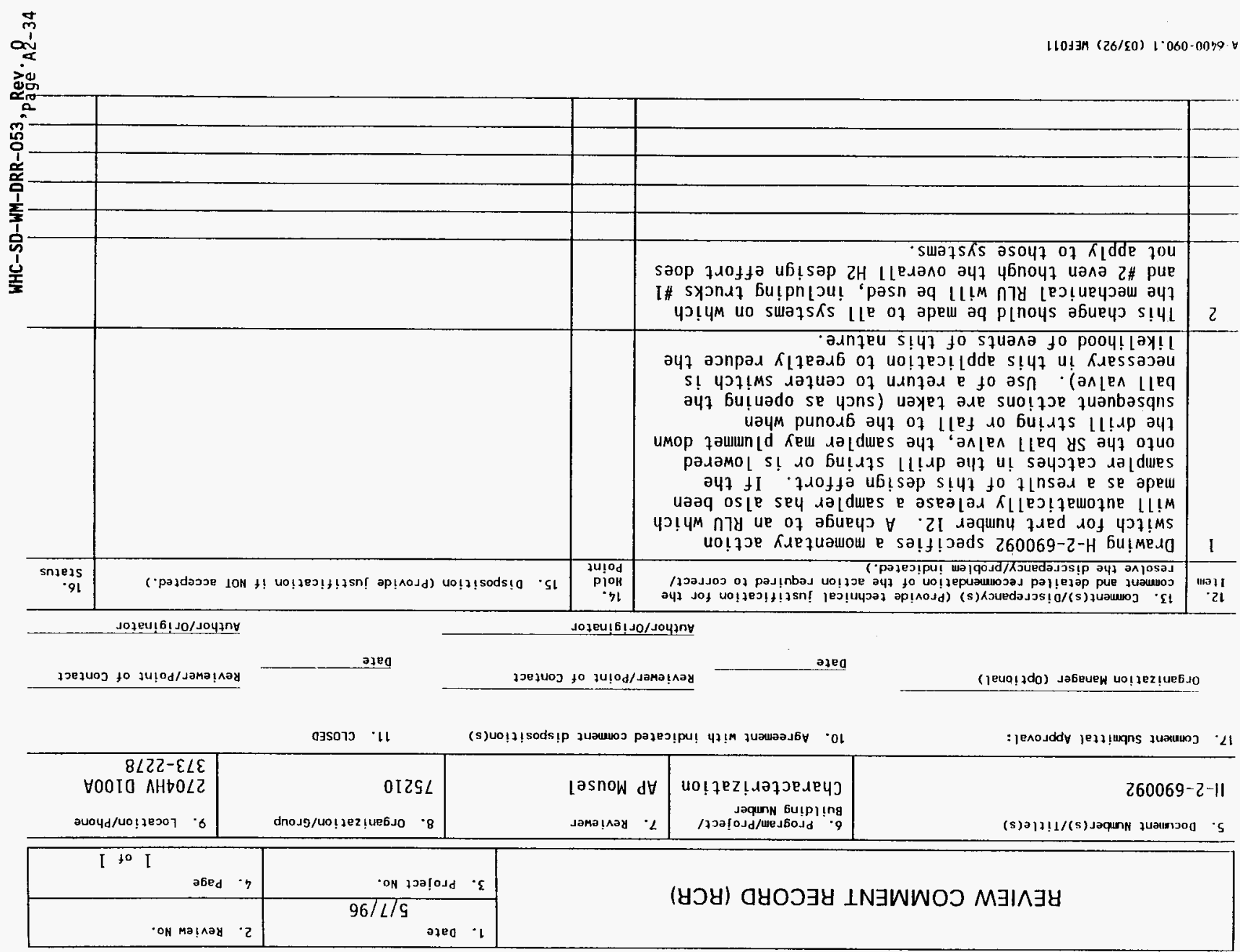


WHC-SD-WM-DRR-053, Rev. 0

Page A3-1

\section{ATTACHMENT 3}

Meeting Minutes 



\section{MEETING MINUTES}

SUBJECT: Final Design Review Briefing, RMCST Modifications for Flammable Gas Tanks

\begin{tabular}{|c|c|c|c|c|}
\hline \multicolumn{2}{|l|}{$\begin{array}{l}\text { TO: } \\
\text { Distribution }\end{array}$} & \multicolumn{3}{|c|}{$\begin{array}{l}\text { BUILDING } \\
\text { N/A }\end{array}$} \\
\hline $\begin{array}{l}\text { FROM: } \\
\text { J. E. Corbett }\end{array}$ & & $\begin{array}{l}\text { CHAIR: } \\
\text { R. J }\end{array}$ & nchard & \\
\hline $\begin{array}{l}\text { OEPARTMENT-OPERATION-COMPONENT } \\
\text { Characterization }\end{array}$ & $\begin{array}{l}\text { AREA } \\
200 E\end{array}$ & $\begin{array}{l}\text { SHIFT } \\
\text { Day }\end{array}$ & $\begin{array}{l}\text { DATE OF MEETING } \\
2 / 6 / 96\end{array}$ & $\begin{array}{l}\text { NUMBER ATIENDING } \\
\text { See Roster }\end{array}$ \\
\hline
\end{tabular}

The meeting was chaired by Roy Blanchard. A list of attendees is contained on the attached meeting roster. The meeting opened with introductions and discussion of objectives and responsibilities. Adjustments were made to the design review committee. The committee assignments agreed to are as follows:

RJ BLANCHARD
JE CORBETT
PJ MARTELL
JA HARVEY
ME HUDA
LS KROGSRUD
NJ MILLIKEN
DC BOARD
ML. MCELROY
GN BOECHLOR
WF WHITE
AP MOUSEL
GJ BOGEN
FA SCHMORDE
BJ SHOEMAKE
JL SMALLEY
JD CRIDDLE

DESIGN REVIEW CHAIRMAN

DESIGN REVIEW SECRETARY

ENVIRONMENTAL ENGINEERING

INDUSTRIAL SAFETY

INDUSTRIAL SAFETY - ALTERNATE

NUCLEAR SAFETY

SAFETY ANALYSIS

QUAL ITY ASSURANCE

QUALITY ASSURANCE - ALTERNATE

MECHANICAL ENGINEERING

ELECTRICAL ENGINEERING

FIELD ENGINEER ING

OPERATIONS

OPERATIONS - ALTERNATE

MAINTENANCE

COGNIZANT DESIGN ENGINEER

COGNIZANT ELECTRICAL DESIGN ENGINEER

The Chairman explained that the Safety Assessment for RMCS in flammable gas tanks is under development and the open issues being resolved are responsible for the recent rescheduling of the design review briefing. When the SA controls are finalized a compliance matrix will be sent out to committee members for review.

Jeff Smalley presented the mechanical portion of the design presentation which consists of 12 Engineering Change Notices (ECN's) to the current RMCS design, and drawings/sketches of the new RLU, riser adapter, riser sleeve, and riser sleeve spray washer. The design information for these components was provided for information only, and will be finalized in approximately 10 days, and will then be formally submitted to committee members for review.

Jim Criddle presented the electrical portion of the design presentation which consists of 3 ECN's to the current RMCS design. A drawing package was also included, showing the ECN's incorporated. Although changes to the Programmable Logic Controller (PLC) are anticipated, this information is not currently defined. When the SA controls are finalized, ECN's related to the PLC will be formally submitted to committee members for review.

John Corbett handed out the design review checklist to be used. Committee members present agreed to the checklist, provided comments are incorporated. The revised checklist will be sent out with these minutes. At the end of the meeting, reviewers were asked to submit RCR's to John Corbett, within 10 working days. The next meeting is scheduled for $2 / 20 / 96$.

LISTING OF ECN'S FOR REVIEW Mechanical:628706 through 628715, 628717, and 628720 . Electrical:626740 through 626742 . 
FINAL DESIGN REVIEW

WHC-SD-WM-DRR-DS2, Rev. 0

-053 Page A3-3

Rotary Mode Core Sampling Truck Modifications for Flammable Gas Tanks

\section{MEETING ROSTER}

SUBנEct: $100 \%$ Design Review Briefing

DATE: $2 / 6 / 96$

LOCATION: $2704 \mathrm{HV} / \mathrm{G} 229$

CHAIRMAN: R. J. Blanchard

Program: Characterization

\begin{tabular}{|c|c|c|c|}
\hline NAME & COMPANY & MSIN & PHONE \\
\hline dotn Corbet & WHC & $57-12$ & $2-2001$ \\
\hline BE BOANAMAED & (1) & $57-12$ & $3-1244$ \\
\hline IDON BOARD be & $\cdots$ & $\vdots \quad \ldots \ldots$ & - \\
\hline Glucian Flubte & WHC & $52-\phi 2$ & 376.8925 \\
\hline GREG I BOCEN & WHC & $37-12$ & $373-5822$ \\
\hline 1)emis temilto & NHC & $5 \cdots-2$ & $372-1130$ \\
\hline John Martill & $w i+c^{3}$ & 53.95 & 373.5417 \\
\hline$\therefore \because E n: 1100$ & $\angle A T A$ & 37 & $3>3-3728$ \\
\hline (1), ty k 165 & WHC & $5>01$ & $2-1667$ \\
\hline & & & \\
\hline & & & \\
\hline & & & \\
\hline & & & \\
\hline & & & \\
\hline & & & \\
\hline & & & \\
\hline & & & \\
\hline & & & \\
\hline & & & \\
\hline & & & \\
\hline & & & \\
\hline & & & \\
\hline & & & \\
\hline & & & \\
\hline
\end{tabular}




\section{MEETING MINUTES}

suBuEct: Final Design Review Briefing, RMCST Modifications for Flammable Gas Tanks

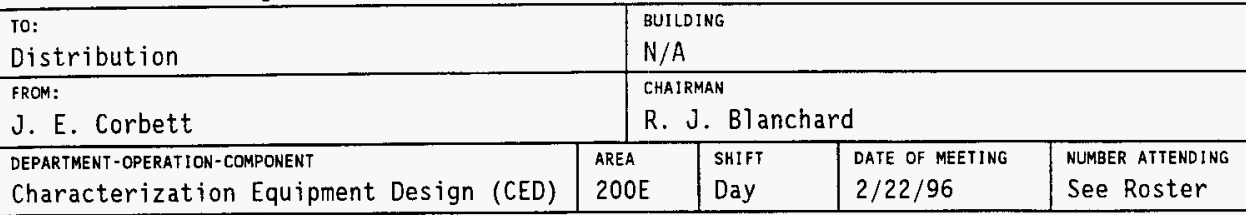

The meeting was chaired by Roy Blanchard. A list of attendees is contained on the attached meeting roster. The meeting opened with introductions and discussion of the design review scope and objectives, and the need for multiple briefings to accommodate the design schedule.

The first order of business was to complete the design review process for the ECN's that had been submitted for review at the February 6, 1996 meeting. There were a total of 18 RCR's received. Jim Criddle gave a verbal report of the dispositions for the four electrical related RCR's. Copies will be provided to anyone who requests one (all RCR's will be included in the final design review report). Jeff Smalley reported that one RCR was considered out of scope, as it requested a change to existing hose reel labeling that is not directly related to modifications for flammable gas tank sampling. Andy Mousel (author of the RCR) and CED later agreed that the request would be considered outside the formal design review process. Jeff handed out copies of the remaining 13 RCR's, which are signed off as closed.

Roy Blanchard reported on the status of the Bureau of Mines testing (the only ignitions thus far are attributed to heat buildup, during drilling beyond the operating envelope, and not sparking). Dennis Hamilton noted that the test environment is worst case, as the oxygen-hydrogen gas mixture is the bounding condition for ignition.

The second portion of the meeting was a briefing on the new RLU and riser equipment. Jeff Smalley handed out the drawings for review. Committee members were asked to have comments submitted, via RCR to John Corbett, within 10 working days. The purpose of the riser liner was described by Jeff as twofold: to allow additional downforce to be used during push mode sampling, and to provide a sleeve to suppress sparking during rotary mode sampling of flammable gas tanks.

The status of the SA was also addressed. LANL is to deliver the "final draft" to WHC on Saturday, February 24th. Changes to the Programmable Logic Controller (PLC) wi11 be baselined on the SA controls from the final draft. Software documentation and ECN'S related to the PLC will be formally submitted to committee members for review. CED will develop a compliance matrix based on the SA controls, including any changes made before the SA is released and approved.

The next meeting is scheduled for Wednesday, March 6th, at 1 PM.

\section{LISTING OF DRAWINGS HANDED OUT}

FOR REVIEW: H-2-690128, H-2-690131, H-2-690132, and H-2-690142

FOR INF0: Unreleased RMCST H-2-6900xx drawings, Revised to include flammable gas tank ECN information; $\mathrm{H}-2-690000,01,05,09,12,15,16,20,26-28,30$, $35,55, \& 90$. 


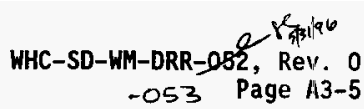

FINAL DESIGN REVIEW

Rotary Mode Core Sampling Truck Modifications for Flammable Gas Tanks

\section{MEETING ROSTER}

SUBJECT: $100 \%$ Design Review Briefing II

\begin{tabular}{|l|l|}
\hline Date $2 / 22 / 96$ & chatrman: R. J. Blanchard \\
\hline Location: $2704 \mathrm{HV} /$ G227 & program: Characterization
\end{tabular}

\begin{tabular}{|c|c|c|c|}
\hline NAME & COMPANY & MSIN & PHONE \\
\hline DON CORBET & $\omega+c$ & $57-02$ & $2-2001$ \\
\hline$\exists \Delta$ CeIDOCE Sr. & WHC & $57-12$ & $6-9224$ \\
\hline DW Namitton & wH $C$ & $57-12$ & $2-1130$ \\
\hline 65 BOCEN & $\omega_{H C}$ & $57-01$ & $3-5822$ \\
\hline M.L. MSELEOL & WHC & $57-07$ & $3-5588$ \\
\hline 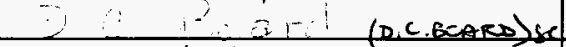 & $1+1+1$ & $37-67$ & 3.121 \\
\hline Ninim Mullik $6 N$ & WAK & A3-37 & $376-7846$ \\
\hline FRED SCHMORDE & WHC & 57.01 & $376-0662$ \\
\hline Eturitiva & WHC & $57-12$ & $3-1340$ \\
\hline 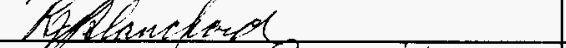 & WAAC & S>-P & $3=1243$ \\
\hline Het Hoechle & $W / B<$ & Ri17 & 3304 \\
\hline & & & \\
\hline & & & \\
\hline & & & \\
\hline & & & \\
\hline & & & \\
\hline & & & \\
\hline & & & \\
\hline - & & & \\
\hline & & & \\
\hline & & & \\
\hline & & & \\
\hline & & & \\
\hline & & & \\
\hline
\end{tabular}




\section{MEETING MINUTES}

SUbJect: Final Design Review Closeout, RMCST Modifications for Flammable Gas Tanks

\begin{tabular}{|c|c|c|c|c|}
\hline TO: & \multicolumn{4}{|c|}{ BUILDING } \\
\hline Distribution & \multicolumn{4}{|c|}{$N / A$} \\
\hline $\begin{array}{l}\text { FROM: } \\
\text { J. E. Corbett }\end{array}$ & \multicolumn{4}{|c|}{$\begin{array}{l}\text { CHAIRMAN } \\
\text { R. J. Bl anchard }\end{array}$} \\
\hline $\begin{array}{l}\text { DEPARTMENT-OPERATION-COMPONENT } \\
\text { Characterization Equipment Design (CED) }\end{array}$ & $\begin{array}{l}\text { AREA } \\
\text { 200E }\end{array}$ & $\begin{array}{l}\text { SHIFT } \\
\text { Day }\end{array}$ & $\begin{array}{l}\text { DATE OF MEETING } \\
4 / 25 / 96\end{array}$ & $\begin{array}{l}\text { MUMBER ATTENDING } \\
\text { See Roster }\end{array}$ \\
\hline
\end{tabular}

The meeting was chaired by Roy Blanchard. A list of attendees is contained on the attached meeting roster. The meeting opened with introductions and discussion of the process required to close out the design review.

Jim Criddle and Galen Wilson discussed the SA controls compliance matrix. Each credited design feature 1 isted in chapter six of the SA was read and, for all features not covered by the original design of trucks $3 \& 4$, the related flammable gas mod ECN or drawing number was noted. A design change, to satisfy the requirement for footclamp closure by two independent actions, is in work and is not currently available for review.

The rest of the meeting followed the attached agenda, with the following ECN's approved for release: 628706 through $628715,628717,628720$, and 626740 through 626742 . The following new drawings were approved for release: H-2-690128, H-2-690131, H-2-690132, and $\mathrm{H}-2-690142$. All RCR's related to these drawings and $E C N^{\prime} s$ were closed. The design review checklist, with dispositions and comments made during the meeting, is attached.

In closing remarks, it was noted that the ATP/OTP and Bureau of Mines testing should be carried as open items for the design review. Nancy Milliken noted that the controls listed in the SA for RMCS in flammable gas tanks would have to be followed for RMCS in any tank, unless SARR-031 is updated to reflect new information on envelope testing. [Although a change in SARR-031 is beyond the scope of this design review, it is an important concern for future sampling.]

The new material handed out for review included two additional ECN's (623775 \& 630017) and two new drawings (H-2-690134 \& H-2-690136). Due to organizational changes, Kathy Tollefson will now be responsible for the environmental review and Omar Jaka will be responsible for the nuclear safety review. Dan Owen is representing the maintenance organization for Bill Shoemeke. Galen Wilson is the cognizant mechanical design engineer. Comments, in the form of RCR's sent to John Corbett, are due by $5 / 2 / 96$. The design review closeout for this material is scheduled for $5 / 7 / 96,1 \mathrm{PM}$. 


\section{FINAL DESIGN REVIEW \\ Rotary Mode Core Sampling Truck Modifications for Flammable Gas Tanks}

\section{AGENDA}

FINAL DESIGN REVIEW CLOSEOUT MEETING

- Background/Introductions

- ECN's for Approval

- New Drawings for Approval

- Design Review Checklist

- Presentation of New Material for Review

- Closing Remarks 
FORMAL DESIGN REVIEW CHECKLIST

Rotary Mode Core Sampling Truck Modifications for Flammable Gas Tanks

Documents Reviewed: $\quad$ ECN\# 628706 through $628715,628717,628720, \& 626740$ through 626742 ;

H-2 drawing\# 690128, 690131, 690132, \& 690142,

and WHC-SD-WM-DRR-052 REV. 0.

\begin{tabular}{|c|c|c|c|c|c|}
\hline Item & Review Consideration & Yes & No & NA & Remarks \\
\hline 1 & $\begin{array}{l}\text { Have assumptions necessary to perform the design } \\
\text { task been adequately described and are they } \\
\text { reasonable? Where necessary, have assumptions been } \\
\text { identified for reverification when the design task has } \\
\text { been completed? }\end{array}$ & $\mathrm{x}$ & & & Assumplions were conservative. \\
\hline 2 & $\begin{array}{l}\text { Have the appropriate Quality Assurance requirements } \\
\text { been specified? }\end{array}$ & $\mathrm{x}$ & & & \\
\hline 3 & Were sources of information identified? & $\mathbf{X}$ & & & \\
\hline 4 & $\begin{array}{l}\text { Does the design meet the established requirements or } \\
\text { design criteris? }\end{array}$ & $\mathrm{x}$ & & & \\
\hline 5 & $\begin{array}{l}\text { Does the design meet established requirements for } \\
\text { associated system physical and functional interfaces? }\end{array}$ & $\mathrm{x}$ & & & \\
\hline 6 & Are there any interface problems? & $\mathrm{x}$ & & & \\
\hline 7 & $\begin{array}{l}\text { Has appropriate consideration been given to use of } \\
\text { standardized parts, materials and processes, and have } \\
\text { engineering standards and criteria been specified } \\
\text { properly in the design? }\end{array}$ & $\mathrm{x}$ & & & \\
\hline 8 & $\begin{array}{l}\text { Does the design represent the simplest design } \\
\text { consistent with functional requirements and expected } \\
\text { service conditions? }\end{array}$ & & & $\mathrm{x}$ & $\begin{array}{l}\text { Not reviewed, due to schedule } \\
\text { constraints. }\end{array}$ \\
\hline 9 & $\begin{array}{l}\text { Can the equipment be readily assembled/disassembled } \\
\text { as designed? }\end{array}$ & $\mathrm{x}$ & & & \\
\hline 10 & $\begin{array}{l}\text { Does the design minimize overall cost to the extent } \\
\text { practicable? }\end{array}$ & & & $\mathrm{x}$ & $\begin{array}{l}\text { Not reviewed, due to schedule } \\
\text { constraints. }\end{array}$ \\
\hline 11 & $\begin{array}{l}\text { Are the specified materials compatible with each } \\
\text { other and the environmental conditions to which the } \\
\text { material will be exposed? }\end{array}$ & $\mathrm{x}$ & & & \\
\hline 12 & $\begin{array}{l}\text { Are the applicable codes, standards and requirements. } \\
\text { including revisions, properly identified and are their } \\
\text { design requirements provided for? }\end{array}$ & $\mathrm{x}$ & & & \\
\hline 13 & $\begin{array}{l}\text { Have modifications to cammercial grade items and } \\
\text { any associated verification operations or tests been } \\
\text { appropriately documented? }\end{array}$ & $\mathbf{x}$ & & & \\
\hline 14 & Have qualified and certified parts been specified? & $\mathrm{x}$ & & & \\
\hline
\end{tabular}




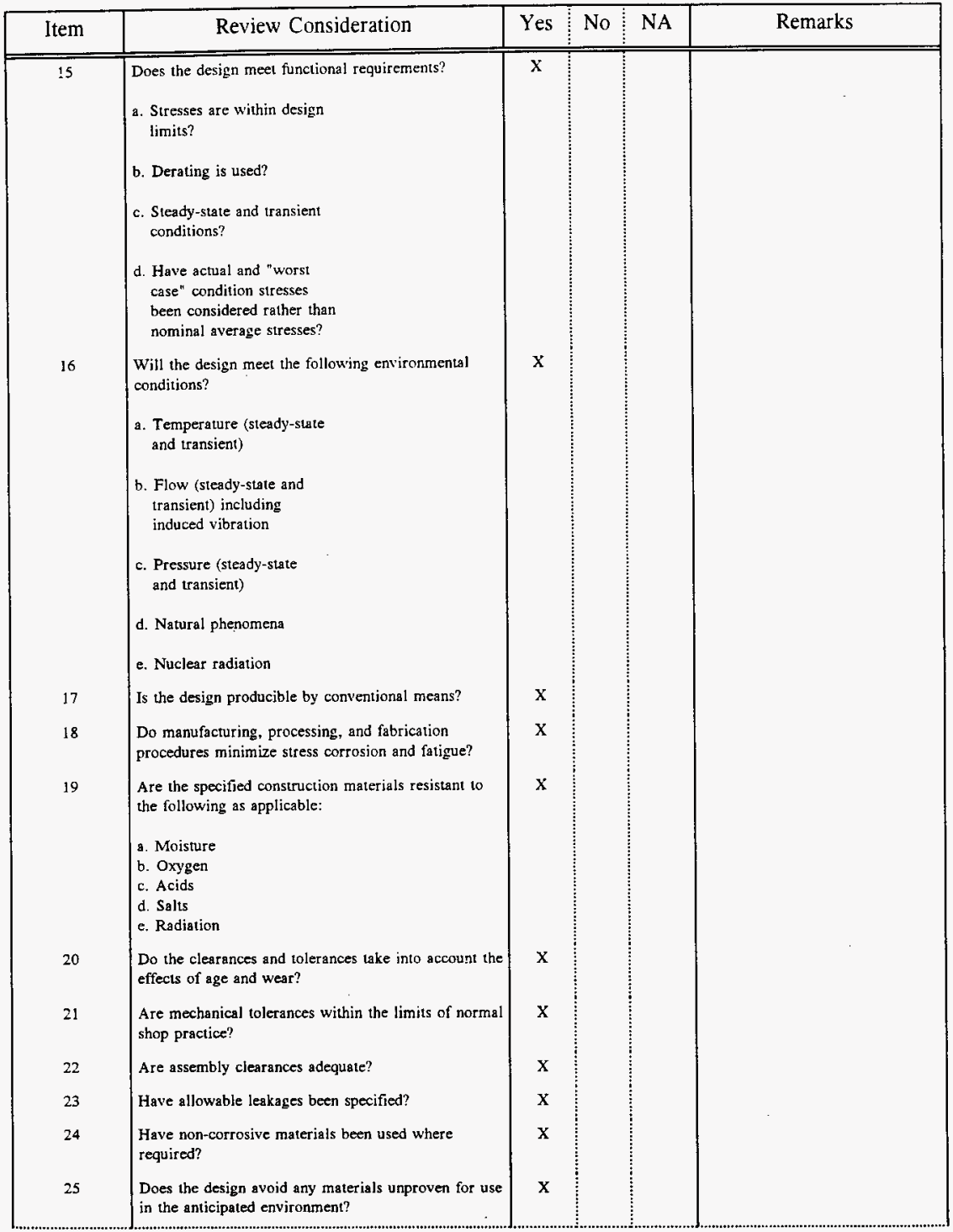




\begin{tabular}{|c|c|c|c|c|c|}
\hline Item & Review Consideration & Yes & No & $\overline{\mathrm{NA}}$ & Remarks \\
\hline 26 & $\begin{array}{l}\text { Can the assembly be stored for extended periods of } \\
\text { time without degrading effects? }\end{array}$ & $\mathrm{x}$ & & & $\begin{array}{l}\text { Applied to equipment required to } \\
\text { enter the tank. }\end{array}$ \\
\hline 27 & $\begin{array}{l}\text { Has the design appropriately considered maintenance, } \\
\text { operation and reliability, including maintenance } \\
\text { procedures and techniques, unique maintenance } \\
\text { requirements and frequencies? }\end{array}$ & $x$ & & & \\
\hline 28 & $\begin{array}{l}\text { Are costings (or finishes) compatible with the } \\
\text { expected environment? With expected usage? }\end{array}$ & $\mathrm{X}$ & & & \\
\hline 29 & $\begin{array}{l}\text { Are surface finish requirements the least stringent } \\
\text { possible? }\end{array}$ & $\mathrm{X}$ & & & \\
\hline 30 & $\begin{array}{l}\text { Are required tolerances, fabrication techniques, } \\
\text { processes, etc., consistent with standard practices? }\end{array}$ & $\mathrm{X}$ & & & \\
\hline 31 & $\begin{array}{l}\text { Can the design and its parts be easily inspected for } \\
\text { conformance to engineering specifications? }\end{array}$ & $\mathrm{X}$ & & & \\
\hline 32 & $\begin{array}{l}\text { Has adequate accessibility been provided for in- } \\
\text { service inspection? }\end{array}$ & $\mathrm{X}$ & & & \\
\hline 33 & $\begin{array}{l}\text { Does the design meet all established safety } \\
\text { requirements? }\end{array}$ & $\mathrm{x}$ & & & \\
\hline 34 & $\begin{array}{l}\text { Has an acceptable level of radiation exposure been } \\
\text { defined? }\end{array}$ & $\mathrm{X}$ & & & . \\
\hline 35 & $\begin{array}{l}\text { Have personnel radiation protection requirements } \\
\text { been considered and identified? }\end{array}$ & $\mathrm{x}$ & & & \\
\hline 36 & $\begin{array}{l}\text { Have nuclear criticality safety considerations been } \\
\text { incorporated? }\end{array}$ & & & $x$ & None required. \\
\hline 37 & $\begin{array}{l}\text { Have necessary features been provided to mainiain } \\
\text { personnel radiation exposure as low as reasonably } \\
\text { achievable? }\end{array}$ & $\mathbf{x}$ & & & \\
\hline 38 & $\begin{array}{l}\text { Can the hardware be adequately disposed of after use } \\
\text { if it is radiologically or chemically contaminated? }\end{array}$ & $x$ & & & $\begin{array}{l}\text { Note: The riser sleeve is in } \\
\text { sections. }\end{array}$ \\
\hline 39 & $\begin{array}{l}\text { Have requirements for storing the equipment been } \\
\text { defined? }\end{array}$ & & & $\mathbf{x}$ & Equipment is mobile. \\
\hline 40 & $\begin{array}{l}\text { Have adequate acceptance criteria been specified and } \\
\text { are the verification methods stated appropriately? }\end{array}$ & $X$ & & & \\
\hline 41 & $\begin{array}{l}\text { Have welding, bolting, joining methods been } \\
\text { adequately specified? }\end{array}$ & $\mathrm{x}$ & & & \\
\hline 42 & Have NDE methods been applied correctly? & $\mathrm{x}$ & & & \\
\hline 43 & $\begin{array}{l}\text { Will a separate Acceptance Test Spec/Procedure be } \\
\text { required? } \\
\text { If yes, identify responsible organization(s) } \\
\text { for preparation and issue (TBD if unknown) }\end{array}$ & $\mathbf{X}$ & & & $\begin{array}{l}\text { Characterization Equipment } \\
\text { Design will provide ATP's. }\end{array}$ \\
\hline 44 & $\begin{array}{l}\text { Have human factors engineering and operability been } \\
\text { considered? }\end{array}$ & $x$ & & & \\
\hline 45 & $\begin{array}{l}\text { Is an Operation and Maintenance Manual required? } \\
\text { If so, have requirements been clearly identified? }\end{array}$ & & $X$ & & $\begin{array}{l}\text { Operating and maintenance } \\
\text { procodures exist. Characterization } \\
\text { Field Engineering will update. }\end{array}$ \\
\hline
\end{tabular}




\begin{tabular}{|c|c|c|c|c|c|}
\hline Item & Review Consideration & Yes & No & NA & Remarks \\
\hline 46 & $\begin{array}{l}\text { Are current operating documents (procedures, } \\
\text { specifications, etc.) applicable to the design or are } \\
\text { changes necessary? }\end{array}$ & & $\mathrm{x}$ & & $\begin{array}{l}\text { Characterization Field Engineering } \\
\text { will provide documents. }\end{array}$ \\
\hline 47 & $\begin{array}{l}\text { Does the design use engineered safety and operational } \\
\text { protections to avoid an excessive risk-taking } \\
\text { dependence on administrative infallibility? }\end{array}$ & $\mathrm{X}$ & & & \\
\hline 48 & $\begin{array}{l}\text { Are reliability requirements specified? If so, does the } \\
\text { reliability analysis of the design meet the specified } \\
\text { reliability requirements? }\end{array}$ & $\mathrm{X}$ & & & Information is in the SA. \\
\hline 49 & $\begin{array}{l}\text { Have all credible non-standard conditions been } \\
\text { properly considered? }\end{array}$ & $\mathrm{X}$ & & & \\
\hline 50 & Is the equipment, system, or facility operable? & $\mathrm{X}$ & & & $\begin{array}{l}\text { This will be demonstrated by } \\
\text { OTP. }\end{array}$ \\
\hline 51 & $\begin{array}{l}\text { Is the equipment design adequate to implement the } \\
\text { proposed maintensnce philosophy? }\end{array}$ & $\mathrm{x}$ & & & \\
\hline 52 & $\begin{array}{l}\text { If any development work is needed, has it been } \\
\text { funded or performed? }\end{array}$ & $\mathrm{x}$ & & & \\
\hline 53 & Has drawing traceability been provided? & $\mathrm{x}$ & & & \\
\hline 54 & $\begin{array}{l}\text { Has the need for safety analysis of this design been } \\
\text { determined by Safety? }\end{array}$ & $\mathrm{X}$ & & & \\
\hline 55 & $\begin{array}{l}\text { Is the equipment, system, or facility covered by an } \\
\text { existing Safety Analysis Report? If not, complete the } \\
\text { safety analysis in time to incorporate findings of the } \\
\text { analyzed in the design. }\end{array}$ & $\mathrm{X}$ & & & $\begin{array}{l}\text { SA is WHC-approved, DOE } \\
\text { approval in process. }\end{array}$ \\
\hline 56 & $\begin{array}{l}\text { Does the design match the intended (and possible } \\
\text { abnormal) methods of operation of the system or } \\
\text { facility? }\end{array}$ & $\mathbf{x}$ & & & \\
\hline 57 & Is a single point failure analysis required? & $\mathrm{x}$ & & & Completed in the SA. \\
\hline 58 & $\begin{array}{l}\text { Are all indication lights and electrical control } \\
\text { considered fail-safe? }\end{array}$ & $\mathrm{x}$ & & & As required by SA. \\
\hline 59 & $\begin{array}{l}\text { Do the design media, format. content, reproducibility, } \\
\text { and quality comply with all applicable requirements } \\
\text { (including Hanford Plant Standards and referenced } \\
\text { codes and standards)? Are the drawings structured to } \\
\text { meet the needs of users after project completion? }\end{array}$ & $\mathrm{x}$ & & & \\
\hline 60 & $\begin{array}{l}\text { Have availability of power requirements for the } \\
\text { project been verified? }\end{array}$ & $\mathrm{X}$ & & & \\
\hline 61 & $\begin{array}{l}\text { Have requirements for providing as-built drawings } \\
\text { been specified? }\end{array}$ & $\mathrm{X}$ & & & \\
\hline 62 & $\begin{array}{l}\text { Is the design in compliance with applicable regulatory } \\
\text { requirements and/or WHC regulatory commitments? }\end{array}$ & $\mathrm{X}$ & & & \\
\hline 63 & $\begin{array}{l}\text { Are design tolerances appropriate and applied in a } \\
\text { cost-efiective manner and are standard materials and } \\
\text { material sizes used where practicable? }\end{array}$ & $\mathrm{x}$ & & & \\
\hline 64 & $\begin{array}{l}\text { Is all computer software and data properly identified } \\
\text { and controlled? }\end{array}$ & $\mathrm{x}$ & & & \\
\hline
\end{tabular}


WHC-SD-WM-DRR-053, Rev. 0

Page A3-12

FINAL DESIGN REVIEW

Rotary Mode Core Sampling Truck Modifications for Flammable Gas Tanks

\section{MEETING ROSTER}

subject: Final Design Review Closeout Meeting

\begin{tabular}{|l|l|}
\hline Date: $4 / 25 / 96$ & Chairman: R. J. Blanchard \\
\hline LOCATION: $2704 \mathrm{HV} / \mathrm{G} 108 \mathrm{~A}$ & Program: Characterization
\end{tabular}

\begin{tabular}{|c|c|c|c|}
\hline NAME & COMPANY & MSIN & PHONE \\
\hline Galun MP Miks & WHC & $57-12$ & $3-1340$ \\
\hline $\operatorname{lin}-\tau_{i b}=x$ & L.te & $57-08$ & $6-4954$ \\
\hline 1) $6,+1 i) A$ & itte & $5>-27$ & $2-2412$ \\
\hline$M . L M \leq E_{L C O U}$ & WHC & $57-07$ & $3-5588$ \\
\hline 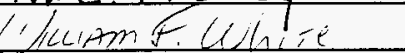 & litic & $H E-11$ & 6.8975 \\
\hline Nanced Millikn & atc & A3-37 & $376-7846$ \\
\hline tP Mouser & $\angle A T A$ & $57-12$ & $373-2278$ \\
\hline 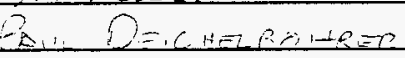 & $w i+c$ & $57-12$ & $3>=-2-37$ \\
\hline BCS DCAMSHAOD & LWPE & $57-12$ & $273-1246$ \\
\hline Sel arasta & wtte & $57-12$ & $376-9224$ \\
\hline Eloxt lor हEा & whe & 57.12 & $372-2001$ \\
\hline & & & \\
\hline & & & \\
\hline & & & \\
\hline & & & \\
\hline & & & \\
\hline & & & \\
\hline & & & \\
\hline & & & \\
\hline & & & \\
\hline & & & \\
\hline & & & \\
\hline & & & \\
\hline
\end{tabular}




\section{MEETING MINUTES}

suBdect: Final Design Review Closeout, RMCST Modifications for Flammable Gas Tanks

\begin{tabular}{|c|c|c|c|c|}
\hline $\begin{array}{l}\text { TO: } \\
\text { Distribution }\end{array}$ & & \multicolumn{3}{|c|}{$\begin{array}{l}\text { BUILDING } \\
\text { N/A }\end{array}$} \\
\hline FROM: & & \multicolumn{3}{|c|}{$\begin{array}{l}\text { CHAIRMAN } \\
\text { R. J. Bl anchard }\end{array}$} \\
\hline $\begin{array}{l}\text { DEPARTMENT-OPERAIION-COMPONENT } \\
\text { Characterization Equipment Design (CED) }\end{array}$ & $\begin{array}{l}\text { AREA } \\
200 E\end{array}$ & $\begin{array}{l}\text { SHIFI } \\
\text { Day }\end{array}$ & $\begin{array}{c}\text { DATE OF MEETING } \\
5 / 7 / 96\end{array}$ & $\begin{array}{l}\text { NUMBER ATTENDING } \\
\text { See Roster }\end{array}$ \\
\hline
\end{tabular}

The meeting was chaired by Roy $\mathrm{Bl}$ anchard, and opened with introductions. Tom Gowin was representing Operations, and Rosa Seda was representing mechanical engineering for Nick Boechler (the meeting roster is attached). Kathy Tollefson sent in a "no comment" RCR to close out the environmental review. The meeting scope was to close-out the design review of the remaining two ECN's and the remaining two new drawings for the RMCST flammable gas mod's. Roy Blanchard reported that the SA was approved by DOE and that the Bureau of Mines testing was essentially complete.

Six RCR's were submitted in support of the meeting scope. The dispositions were discussed and all RCR's were closed. The design committee approved ECN $\# 623775$ \& $\# 630017$ and drawing $\# H-2-690134 \& \# H-2-690136$. There are no open issues in these RCR's.

The design review checklist was revised to include the additional design documents. The completed checklist, including comments made during the meeting, is attached. $*$ There are no open issues in the checklist, except for performing the ATP/OTP and providing procedures.

The SA compliance matrix was reviewed to verify all design features credited in the SA were included in the design review or were part of the existing design. As previously noted in the $4 / 25$ meeting, modifying the footclamp operation is an open item. There are no other open items on the matrix.

In closing remarks, committee members were given the opportunity to raise any other concerns related to the flammable gas mod's. Andy Mousel recommended changing the SR hoist switch to a "return to center" switch, to reduce the possibility of an inadvertent release of the sampler from the RLU. The committee agreed to carry this action as an open item. Mousel agreed to slibmit an RCR to document the required action. The design review closed without further discussion.

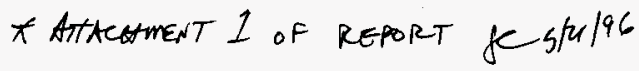




\section{FINAL DESIGN REVIEW \\ Rotary Mode Core Sampling Truck Modifications for Flammable Gas Tanks}

\section{AGENDA}

FINAL DESIGN REVIEW CLOSEOUT MEETING ॥

- Background/Introductions

- RCR Close-out

- ECN and New Drawing Approval

- SA Compliance Matrix

- Design Review Checklist

- Closing Remarks 
FINAL DESIGN REVIEW

Rotary Mode Core Sampling Truck Modifications for Flammable Gas Tanks

\section{MEETING ROSTER}

SUBJEct: Final Design Review Closeout Meeting II

DATE: $5 / 7 / 96$

LOCATION: $2704 \mathrm{HV} / \mathrm{G} 110$

CHairman: R. J. Bl anchard

PROGRAM: Characterization

\begin{tabular}{|c|c|c|c|}
\hline NAME & COMPANY & MSIN & PHONE \\
\hline I.D. CelDols Jr. & $W H C$ & $57-12$ & $376-9224$ \\
\hline AP MOUSEL & $\triangle A T A$ & $57-12$ & $3>3-22>8$ \\
\hline Wiviam F White & $\omega 4 c$ & $46-11$ & $376-8925$ \\
\hline Resolde for GN Boechler & WHC & $45-09$ & $373-0061$ \\
\hline M.L. MSELZOV & Whe & $57-07$ & $373-5588$ \\
\hline OMAR JAKA & Whic & $R 3-2 s$ & $372-2322$ \\
\hline ID. Gowisn & Whe & $53-07$ & $373-0258$ \\
\hline ACY' $\checkmark$ BEANCYHASD & LAHC & $57-, 2$ & $373-124$ \\
\hline town CarbeT & $\omega+c$ & $57-17$ & $372-2001$ \\
\hline in. E.turit & kye & $57-07$ & $372-2412$ \\
\hline & & & \\
\hline & & & \\
\hline & & & \\
\hline & & & \\
\hline & & & \\
\hline & & & \\
\hline & & & \\
\hline & & & \\
\hline & & & \\
\hline & & & \\
\hline & & & \\
\hline & & & \\
\hline & & & \\
\hline & & & \\
\hline
\end{tabular}

